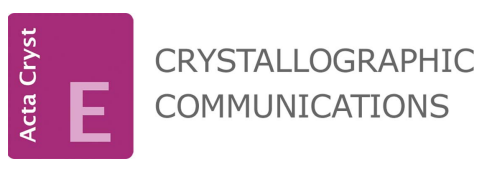

ISSN 2056-9890

Received 16 May 2017

Accepted 22 May 2017

Edited by W. T. A. Harrison, University of Aberdeen, Scotland

Keywords: crystal structure; polymorphism; pyrimethanil; hydrogen bonding.

CCDC reference: 1549998

Supporting information: this article has supporting information at journals.iucr.org/e

\section{A metastable polymorphic form of the antifungal anilinopyrimidine active pyrimethanil}

\author{
Alex R. Eberlin ${ }^{\mathrm{a}}$ and Christopher S. Frampton ${ }^{\mathrm{b} *}$ \\ aJohnson Matthey, Pharmorphix, 250 Cambridge Science Park, Milton Road, Cambridge CB4 OWE, England, and \\ ${ }^{\mathbf{b}}$ Wolfson Centre for Materials Processing, Brunel University London, Kingston Lane, Uxbridge UB8 3PH, England. \\ *Correspondence e-mail: chris.frampton@brunel.ac.uk
}

A second metastable form of the title compound, $\mathrm{C}_{12} \mathrm{H}_{13} \mathrm{~N}_{3}$ (systematic name: 4,6-dimethyl- $N$-phenylpyrimidin-2-amine), was isolated from an attempted cocrystallization experiment with meso-erythriol in dimethyl sulfoxide (DMSO). The crystals of form 2 at $120 \mathrm{~K}$ are monoclinic, space group $P 2_{1} / n$ with $Z^{\prime}=4$ compared to the previously reported triclinic form with $Z^{\prime}=2$ [Sun et al. (2011). Acta Chim. Sin. 69, 1909-1914]. The four independent molecules in the asymmetric unit form two discrete dimeric units through a concerted pair of $\mathrm{N}-$ H $\cdots \mathrm{N}$ hydrogen bonds with a graph-set notation of $R_{2}^{2}(8)$. The origin of the polymorphic behaviour is revealed in that the conformation of each dimer present in the asymmetric unit of the structure is unique and determined by the rotation of the second molecule in the dimer with respect to the first.

\section{Chemical context}

(4,6-Dimethyl-pyrimidin-2-yl)-phenyl-amine, pyrimethanil (1) is a broad spectrum systemic fungicide from the anilinopyrimidine class of agents, which also include cyprodinil and mepanipyrim. It was discovered in 1987 (Buhmann et al., 1988) and is marketed under the trade name SCALA ${ }^{\circledR}$. Anilinopyrimidines are used extensively for protection against leaf moulds and other fungi. In a recent paper (Sun et al., 2011), the synthesis and electronic properties of pyrimethanil were presented, including a discussion on the atomic charges, total energy and frontier orbital energy. As part of this wider study, the crystal structure of pyrimethanil was determined at $295 \mathrm{~K}$ and used as an initial starting model in the structural optimization process. The structure was triclinic, space group $P \overline{1}$, with $Z^{\prime}=2$, with two independent molecules in the asymmetric unit. The two independent molecules form a dimeric structural unit through a concerted pair of $\mathrm{N}-\mathrm{H} \cdots \mathrm{N}$ hydrogen bonds with a graph-set notation of $R_{2}^{2}(8)$. We have recently been investigating the co-crystallization behaviour of pyrimethanil in an attempt to modify the physicochemical properties of the bulk solid material to improve its overall performance. During the course of one of the co-crystallization screens, the crystal structure of a second polymorphic crystal form of pyrimethanil was determined on a crystal that was isolated from the reaction product of an attempted co-crystallization experiment with meso-erythriol in dimethylsulfoxide (DMSO). In this communication, we report the single crystal $\mathrm{X}$-ray structure of this second, metastable, monoclinic polymorphic form of pyrimethanil at $120 \mathrm{~K}$. 


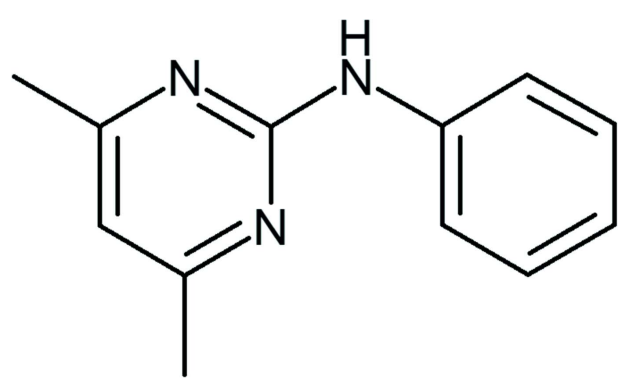

\section{Structural commentary}

The crystal structure of form 2 of pyrimethanil is monoclinic, space group $P 2_{1} / n$ with four independent molecules of pyrimethanil in the asymmetric unit, $\left(Z^{\prime}=4\right)$. For clarity, the independent molecules are labelled with suffixes $A, B, C$ and $D$. The four independent molecules arrange themselves into two dimeric units $A-B$ and $C-D$, each through a concerted pair of $\mathrm{N}-\mathrm{H} \cdots \mathrm{N}$ hydrogen bonds with a graph-set notation of $R_{2}^{2}(8)$, in a similar arrangement to the dimeric structure found in form 1. Figs. 1 and 2 show displacement ellipsoid plots for the two dimers, $A-B$ and $C-D$ and hydrogen-bond distances and angles are given in Table 1 . The phenyl and pyrimidine rings defined by atoms $\mathrm{C} 1-\mathrm{C} 6$ and $\mathrm{N} 2 / \mathrm{N} 3 / \mathrm{C} 7-\mathrm{C} 10$, respectively, for molecules $A$ to $D$ are approximately co-planar. A calculated least-squares plane through the six atoms of the phenyl ring and the six atoms of the pyrimidine ring gave r.m.s. deviations from planarity and a calculated dihedral angle between them as follows: molecule $A, 0.0019 \AA, 0.0050 \AA$, $10.8(1)^{\circ}$; molecule $B, 0.0076 \AA, 0.0102 \AA, 14.8(1)^{\circ}$; molecule $C, 0.0049 \AA, 0.0153 \AA, 8.2(1)^{\circ}$ and molecule $D, 0.0081 \AA$, $0.0105 \AA, 13.5(1)^{\circ}$. The small variation in the angular range of

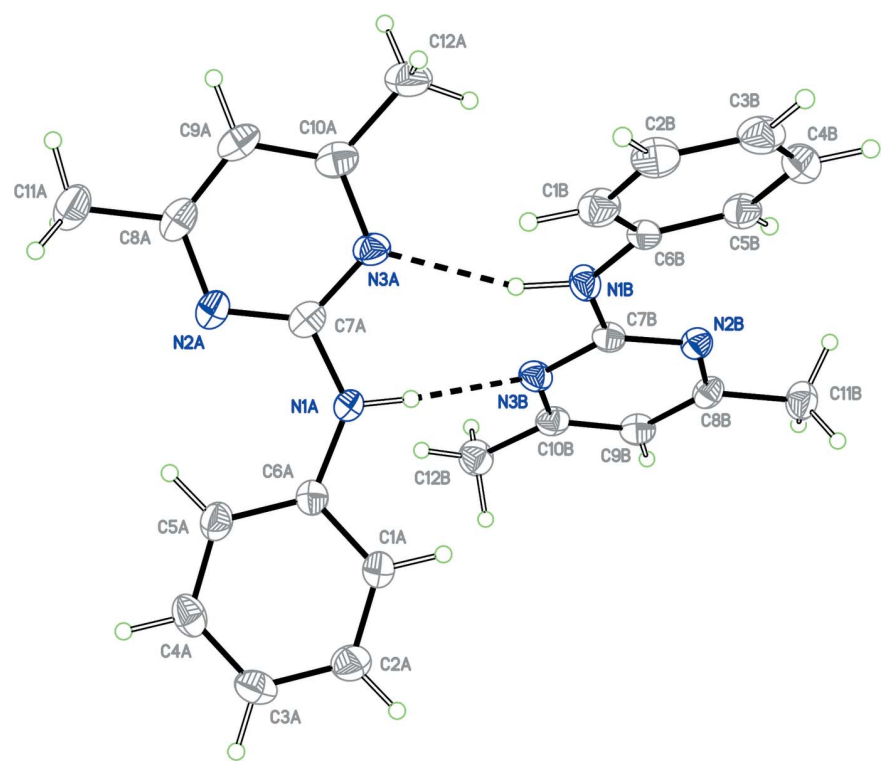

Figure 1

View of the $A-B$ dimer of the asymmetric unit with atom labelling. Ellipsoids are drawn at the $50 \%$ probability level. The intermolecular $\mathrm{N}-\mathrm{H} \cdots \mathrm{N}$ hydrogen bonds are shown as dashed lines.
Table 1

Hydrogen-bond geometry $\left(\AA{ }^{\circ}\right)$.

\begin{tabular}{lllll}
\hline$D-\mathrm{H} \cdots A$ & $D-\mathrm{H}$ & $\mathrm{H} \cdots A$ & $D \cdots A$ & $D-\mathrm{H} \cdots A$ \\
\hline $\mathrm{N} 1 A-\mathrm{H} 1 A B \cdots \mathrm{N} 3 B$ & $0.91(3)$ & $2.11(3)$ & $2.997(3)$ & $165(2)$ \\
$\mathrm{N} 1 B-\mathrm{H} 1 B B \cdots \mathrm{N} 3 A$ & $0.97(3)$ & $2.08(3)$ & $3.022(3)$ & $162(3)$ \\
$\mathrm{N} 1 C-\mathrm{H} 1 C B \cdots \mathrm{N} 3 D$ & $0.94(3)$ & $2.05(3)$ & $2.975(3)$ & $166(3)$ \\
$\mathrm{N} 1 D-\mathrm{H} 1 D B \cdots \mathrm{N} 3 C$ & $0.92(3)$ & $2.08(3)$ & $2.987(3)$ & $167(3)$ \\
\hline
\end{tabular}

the dihedral angles appears consistent with that observed for the other pyrimethanil structures discussed below, 7.5-13.1.

\section{Supramolecular features}

A view of the crystal packing down the $a$-axis is shown in Fig. 3 . The $R_{2}^{2}(8)$ hydrogen-bonded rings defined by atoms $\mathrm{N} 3 A /$ $\mathrm{C} 7 A / \mathrm{N} 1 A / \mathrm{H} 1 A B / \mathrm{N} 3 B / \mathrm{C} 7 B / \mathrm{N} 1 B / \mathrm{H} 1 B B$ and $\mathrm{N} 3 C / \mathrm{C} 7 C / \mathrm{N} 1 C /$ $\mathrm{H} 1 C B / \mathrm{N} 3 D / \mathrm{C} 7 D / \mathrm{N} 1 D / \mathrm{H} 1 D B$ for the two dimers are twisted such that each dimer forms a cross pattern, with a dihedral angle of $42.8(2)^{\circ}$ for dimer $A-B$ and $47.5(2)^{\circ}$ for dimer $C-D$. These dihedral angles are between planes $\mathrm{C} 6 A / \mathrm{N} 1 A / \mathrm{C} 7 A$ and $\mathrm{C} 6 B / \mathrm{N} 1 B / \mathrm{C} 7 B$ for $A-B$ and $\mathrm{C} 6 C / \mathrm{N} 1 C / \mathrm{C} 7 C$ and $\mathrm{C} 6 D / \mathrm{N} 1 D /$ $C 7 D$ for $C-D$. The angles are somewhat reduced in magnitude when compared to the equivalent calculation performed for form 1, $55.7(1)^{\circ}$. Fig. 4 shows an overlay of the two dimeric units in form 2, dimer $A-B$ is shown in violet and $C-D$ in blue, which reveals the origin of the polymorphic behaviour and in turn the reason why $Z^{\prime}=4$. In this figure, molecules $A$ and $C$ have been overlaid (r.m.s. deviation $=0.181 \AA$ ) using the standard routine in Mercury (Macrae et al., 2008). It can be seen that molecule $B$ in the $A-B$ dimer is rotated $134^{\circ}$ with respect to molecule $D$ in the $C-D$ dimer, thus making each dimer unique. It is interesting to note that the dimer found in

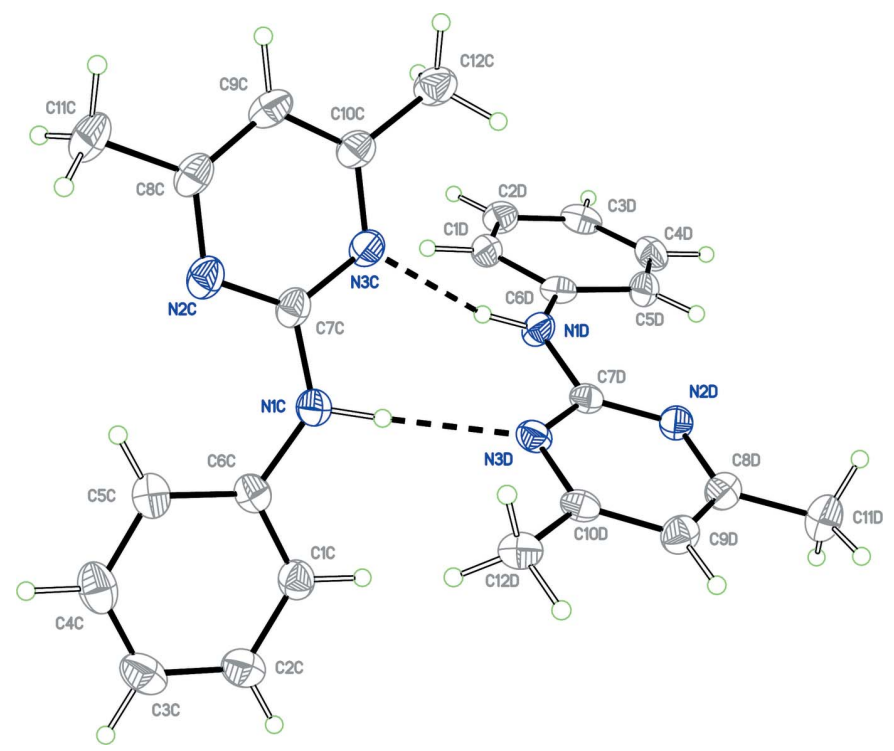

Figure 2

View of the $C-D$ dimer of the asymmetric unit with atom labelling. Ellipsoids are drawn at the $50 \%$ probability level. The intermolecular $\mathrm{N}-\mathrm{H} \cdots \mathrm{N}$ hydrogen bonds are shown as dashed lines. 


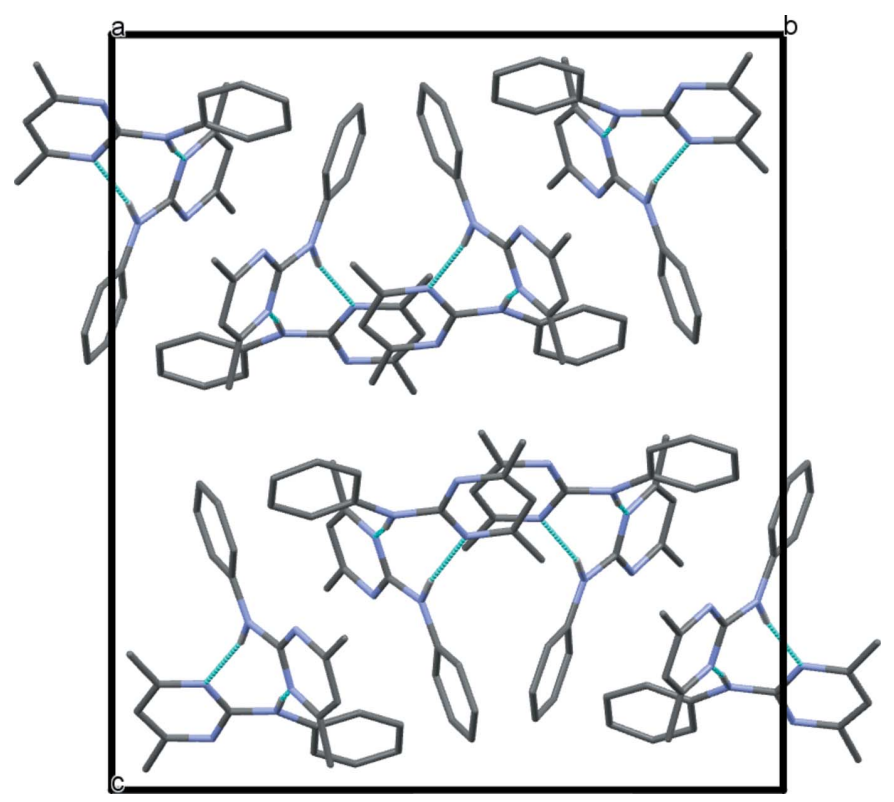

Figure 3

View of the crystal packing down the $a$ axis. Only the nitrogen heteroatom $\mathrm{H}$ atoms are shown for clarity. The intermolecular $\mathrm{N}-$ $\mathrm{H} \cdots \mathrm{N}$ hydrogen bonds (see Table 1 ) are shown as dotted lines.

the structure of form 1 has a similar conformation/orientation to the $C-D$ dimer in the present structure. There are no further significant intermolecular contacts and the crystal packing between dimers appears to be driven largely by van der Waals forces only.

\section{Database survey}

A search of the Cambridge Structural Database (CSD, Version 5.38 update February 2017; Groom et al., 2016) for both the pyrimethanil framework and its protonated counterpart yielded three hits, all of which were genuine examples of the material under investigation. Only one entry was found which related to an example that was not a co-crystal, solvate or salt form and that was for the triclinic, $P \overline{1}, Z^{\prime}=2$, form 1 polymorph (CELNOY; Sun et al., 2011. The remaining two entries were salt forms where the basic nitrogen atom (N3) had been protonated. These examples are the monochloroacetate (MIRYOC; Li et al., 2008) and the p-toluenesulfonate

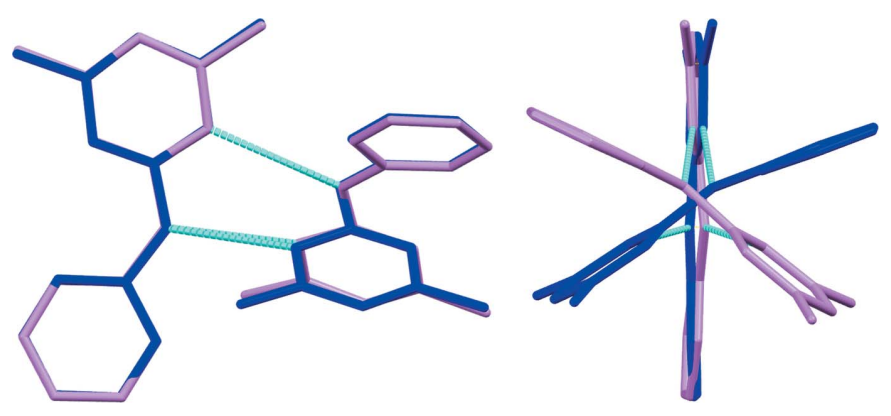

Figure 4

View of the overlay of dimer $A-B$ (violet) and dimer $C-D$ (blue).
Table 2

Experimental details.

\begin{tabular}{|c|c|}
\hline \multicolumn{2}{|l|}{ Crystal data } \\
\hline Chemical formula & $\mathrm{C}_{12} \mathrm{H}_{13} \mathrm{~N}_{3}$ \\
\hline$M_{\mathrm{r}}$ & 199.25 \\
\hline Crystal system, space group & Monoclinic, $P 2_{1} / n$ \\
\hline Temperature $(\mathrm{K})$ & 120 \\
\hline$a, b, c(\AA)$ & $\begin{array}{l}10.5351(4), 19.1686(7), \\
22.1162(8)\end{array}$ \\
\hline$\beta\left(^{\circ}\right)$ & $102.778(4)$ \\
\hline$V\left(\AA^{6}\right)^{3}$ & $4355.6(3)$ \\
\hline$Z$ & 16 \\
\hline Radiation type & Mo $K \alpha$ \\
\hline$\mu\left(\mathrm{mm}^{-1}\right)$ & 0.08 \\
\hline Crystal size $(\mathrm{mm})$ & $0.20 \times 0.15 \times 0.10$ \\
\hline \multicolumn{2}{|l|}{ Data collection } \\
\hline Diffractometer & $\begin{array}{l}\text { Agilent SuperNova, Dual, } \mathrm{Cu} \text { at } \\
\text { zero, Atlas }\end{array}$ \\
\hline Absorption correction & $\begin{array}{l}\text { Multi-scan (CrysAlis PRO; Rigaku, } \\
\text { 2015) }\end{array}$ \\
\hline$T_{\min }, T_{\max }$ & $0.960,1.000$ \\
\hline $\begin{array}{l}\text { No. of measured, independent and } \\
\text { observed }[I>2 \sigma(I)] \text { reflections }\end{array}$ & $16540,7552,4410$ \\
\hline$R_{\text {int }}$ & 0.053 \\
\hline$(\sin \theta / \lambda)_{\max }\left(\AA^{-1}\right)$ & 0.595 \\
\hline \multicolumn{2}{|l|}{ Refinement } \\
\hline$R\left[F^{2}>2 \sigma\left(F^{2}\right)\right], w R\left(F^{2}\right), S$ & $0.056,0.149,1.00$ \\
\hline No. of reflections & 7552 \\
\hline No. of parameters & 565 \\
\hline $\mathrm{H}$-atom treatment & $\begin{array}{l}\mathrm{H} \text { atoms treated by a mixture of } \\
\text { independent and constrained } \\
\text { refinement }\end{array}$ \\
\hline$\Delta \rho_{\max }, \Delta \rho_{\min }\left(\mathrm{e} \AA^{-3}\right)$ & $0.24,-0.29$ \\
\hline
\end{tabular}

Computer programs: CrysAlis PRO (Rigaku, 2015), SHELXD2014 (Sheldrick, 2015), SHELXL2014 (Sheldrick, 2015), SHELXTL (Sheldrick, 2008), Mercury (Macrae et al., 2008) and publCIF (Westrip, 2010).

(XEZFUE; Li et al., 2007). One further example, which is not yet available in the current release of the database, is an exciting 1:1 co-crystal of pyrimethanil with a second antifungal active, dithianon (SAJJAR; Pöppler et al., 2017). This material is currently being marketed under the trade name FABAN ${ }^{\circledR}$.

\section{Synthesis and crystallization}

Crystals of form 2 of pyrimethanil were isolated from the reaction product of an attempted co-crystallization screen with meso-erythriol in dimethylsulfoxide (DMSO). The screen consisted of approximately $20 \mathrm{mg}$ of pyrimethanil being dispensed per vial along with 20 volumes of the appropriate solvent, approx. $400 \mu \mathrm{l}$, at room temperature. The appropriate coformer (ratio 1:1) was also dispensed into the vials in the same manner along with a further 20 volumes of solvent. For the vials that gave clear solutions, these were filtered through a $4 \mu \mathrm{m}$ filter to remove any potential seeds that may remain in the solution. The vials were placed in a platform shaker incubator (Heidolph Titramax/Inkubator 1000) and subjected to a series of heating-cooling cycles under shaking from room temperature (RT) to $323 \mathrm{~K}$ ( $8 \mathrm{~h}$ cycles; heating to $323 \mathrm{~K}$ for $4 \mathrm{~h}$ and then cooling to RT for a further $4 \mathrm{~h}$ ) for a maximum of $48 \mathrm{~h}$. The resulting solutions were then allowed to evaporate slowly over a period of 14 days. The solid materials obtained 
from the screen were analysed by X-ray powder diffraction and were investigated further if they displayed diffraction patterns that were clearly different from that of form 1 or the coformer itself. Unfortunately, it has not been possible thus far to repeat the above experiment to generate more form 2 material, leading us to conclude that form 2 is a metastable form with respect to form 1 .

\section{Refinement}

Crystal data, data collection, and structure refinement details are summarized in Table 2. The positional coordinates of the $\mathrm{N}$-bound $\mathrm{H}$ atoms were all located from a Fourier-difference map and freely refined. All the remaining $\mathrm{H}$ atoms were placed geometrically in idealized positions and refined using a riding model (including free rotation about the methyl $\mathrm{C}-\mathrm{C}$ bond), with $\mathrm{C}-\mathrm{H}=0.95-0.99 \AA$ and $U_{\text {iso }}=1.5 U_{\text {eq }}(\mathrm{C})$ for methyl groups and $1.2 U_{\mathrm{eq}}(\mathrm{C})$ for other $\mathrm{H}$ atoms.

\section{References}

Buhmann, U., Westermann, J., Baumert, D., Pieroh, E., Cliff, G. R. \& Richards, I. C. (1988). US Patent US4783459.

Groom, C. R., Bruno, I. J., Lightfoot, M. P. \& Ward, S. C. (2016). Acta Cryst. B72, 171-179.

Li, J.-C., Feng, Y.-H., Lin, Q. \& Zhang, D.-L. (2007). Acta Cryst. E63, o1162-o1163.

Li, J.-C., Qiu, X.-Q., Feng, Y.-H. \& Lin, Q. (2008). Acta Cryst. E64, 0318.

Macrae, C. F., Bruno, I. J., Chisholm, J. A., Edgington, P. R., McCabe, P., Pidcock, E., Rodriguez-Monge, L., Taylor, R., van de Streek, J. \& Wood, P. A. (2008). J. Appl. Cryst. 41, 466-470.

Pöppler, A.-C., Corlett, E. K., Pearce, H., Seymour, M. P., Reid, M., Montgomery, M. G. \& Brown, S. P. (2017). Acta Cryst. C73, 149156.

Rigaku (2015). CrysAlis PRO. Rigaku Oxford Diffraction, Oxfordshire, England.

Sheldrick, G. M. (2008). Acta Cryst. A64, 112-122.

Sheldrick, G. M. (2015). Acta Cryst. C71, 3-8.

Sun, X.-H., Li, J., Liu, Y. \& Ma, H.-X. (2011). Acta Chim. Sin. 69, 1909-1914.

Westrip, S. P. (2010). J. Appl. Cryst. 43, 920-925. 


\section{supporting information}

Acta Cryst. (2017). E73, 886-889 [https://doi.org/10.1107/S2056989017007563]

\section{A metastable polymorphic form of the antifungal anilinopyrimidine active pyrimethanil}

\section{Alex R. Eberlin and Christopher S. Frampton}

Computing details

Data collection: CrysAlis PRO (Rigaku, 2015); cell refinement: CrysAlis PRO (Rigaku, 2015); data reduction: CrysAlis PRO (Rigaku, 2015); program(s) used to solve structure: SHELXD2014 (Sheldrick, 2015); program(s) used to refine structure: SHELXL2014 (Sheldrick, 2015); molecular graphics: SHELXTL (Sheldrick, 2008) and Mercury (Macrae et al., 2008); software used to prepare material for publication: SHELXTL (Sheldrick, 2008), Mercury (Macrae et al., 2008) and publCIF (Westrip, 2010).

4,6-Dimethyl- $N$-phenylpyrimidin-2-amine

Crystal data

$\mathrm{C}_{12} \mathrm{H}_{13} \mathrm{~N}_{3}$

$M_{r}=199.25$

Monoclinic, $P 2_{1} / n$

$a=10.5351$ (4) $\AA$

$b=19.1686(7) \AA$

$c=22.1162(8) \AA$

$\beta=102.778(4)^{\circ}$

$V=4355.6(3) \AA^{3}$

$Z=16$

Data collection

Agilent SuperNova, Dual, $\mathrm{Cu}$ at zero, Atlas diffractometer

Radiation source: SuperNova (Mo) X-ray

Source

Mirror monochromator

Detector resolution: 10.5598 pixels $\mathrm{mm}^{-1}$

$\omega$ scans

Absorption correction: multi-scan

(CrysAlis PRO; Rigaku, 2015)

\section{Refinement}

Refinement on $F^{2}$

Least-squares matrix: full

$R\left[F^{2}>2 \sigma\left(F^{2}\right)\right]=0.056$

$w R\left(F^{2}\right)=0.149$

$S=1.00$

7552 reflections

565 parameters

0 restraints
$F(000)=1696$

$D_{\mathrm{x}}=1.215 \mathrm{Mg} \mathrm{m}^{-3}$

Mo $K \alpha$ radiation, $\lambda=0.71073 \AA$

Cell parameters from 2876 reflections

$\theta=2.9-24.5^{\circ}$

$\mu=0.08 \mathrm{~mm}^{-1}$

$T=120 \mathrm{~K}$

Block, colourless

$0.20 \times 0.15 \times 0.10 \mathrm{~mm}$

$T_{\min }=0.960, T_{\max }=1.000$

16540 measured reflections

7552 independent reflections

4410 reflections with $I>2 \sigma(I)$

$R_{\text {int }}=0.053$

$\theta_{\text {max }}=25.0^{\circ}, \theta_{\text {min }}=2.9^{\circ}$

$h=-12 \rightarrow 12$

$k=-22 \rightarrow 18$

$l=-25 \rightarrow 26$

Primary atom site location: structure-invariant direct methods

Hydrogen site location: mixed

$\mathrm{H}$ atoms treated by a mixture of independent and constrained refinement

$w=1 /\left[\sigma^{2}\left(F_{\mathrm{o}}^{2}\right)+(0.055 P)^{2}\right]$

where $P=\left(F_{\mathrm{o}}{ }^{2}+2 F_{\mathrm{c}}{ }^{2}\right) / 3$

$(\Delta / \sigma)_{\max }=0.002$ 
$\Delta \rho_{\max }=0.24$ e $\AA^{-3}$

$\Delta \rho_{\min }=-0.29$ e $\AA^{-3}$

Special details

Geometry. All esds (except the esd in the dihedral angle between two 1.s. planes) are estimated using the full covariance matrix. The cell esds are taken into account individually in the estimation of esds in distances, angles and torsion angles; correlations between esds in cell parameters are only used when they are defined by crystal symmetry. An approximate (isotropic) treatment of cell esds is used for estimating esds involving l.s. planes.

Fractional atomic coordinates and isotropic or equivalent isotropic displacement parameters $\left(\AA^{2}\right)$

\begin{tabular}{|c|c|c|c|c|}
\hline & $x$ & $y$ & $z$ & $U_{\text {iso }} * / U_{\text {eq }}$ \\
\hline N1A & $0.6124(2)$ & $0.75456(11)$ & $0.10435(11)$ & $0.0263(6)$ \\
\hline $\mathrm{H} 1 \mathrm{AB}$ & $0.698(3)$ & $0.7460(13)$ & $0.1205(12)$ & $0.025(7)^{*}$ \\
\hline $\mathrm{N} 2 \mathrm{~A}$ & $0.4761(2)$ & $0.84979(12)$ & $0.06807(10)$ & $0.0261(6)$ \\
\hline $\mathrm{N} 3 \mathrm{~A}$ & $0.6823(2)$ & $0.86154(11)$ & $0.14057(10)$ & $0.0256(5)$ \\
\hline $\mathrm{C} 1 \mathrm{~A}$ & $0.5890(3)$ & $0.63127(14)$ & $0.10191(13)$ & $0.0280(7)$ \\
\hline H1AA & 0.6763 & 0.6288 & 0.1251 & $0.034 *$ \\
\hline $\mathrm{C} 2 \mathrm{~A}$ & $0.5192(3)$ & $0.57049(15)$ & $0.08619(13)$ & $0.0328(7)$ \\
\hline H2AA & 0.5586 & 0.5267 & 0.0988 & $0.039^{*}$ \\
\hline $\mathrm{C} 3 \mathrm{~A}$ & $0.3916(3)$ & $0.57303(15)$ & $0.05197(12)$ & $0.0308(7)$ \\
\hline H3AA & 0.3431 & 0.5314 & 0.0410 & $0.037^{*}$ \\
\hline $\mathrm{C} 4 \mathrm{~A}$ & $0.3367(3)$ & $0.63763(15)$ & $0.03413(12)$ & $0.0304(7)$ \\
\hline H4AA & 0.2497 & 0.6399 & 0.0106 & $0.036^{*}$ \\
\hline $\mathrm{C} 5 \mathrm{~A}$ & $0.4056(2)$ & $0.69886(15)$ & $0.04986(12)$ & $0.0276(7)$ \\
\hline H5AA & 0.3659 & 0.7425 & 0.0372 & $0.033^{*}$ \\
\hline C6A & $0.5331(2)$ & $0.69632(14)$ & $0.08422(12)$ & $0.0234(6)$ \\
\hline C7A & 0.5858 & $0.82446(14)$ & $0.10375(13)$ & $0.0245(7)$ \\
\hline C8A & $0.4627(3)$ & $0.91995(15)$ & $0.06878(13)$ & $0.0289(7)$ \\
\hline C9A & $0.5570(3)$ & $0.96202(15)$ & $0.10415(13)$ & $0.0309(7)$ \\
\hline H9AA & 0.5466 & 1.0113 & 0.1040 & $0.037^{*}$ \\
\hline C10A & $0.6671(3)$ & $0.93098(14)$ & $0.13983(13)$ & $0.0286(7)$ \\
\hline $\mathrm{C} 11 \mathrm{~A}$ & $0.3416(3)$ & $0.94925(16)$ & $0.02772(14)$ & $0.0381(8)$ \\
\hline H11A & 0.2651 & 0.9256 & 0.0364 & $0.057^{*}$ \\
\hline H11B & 0.3446 & 0.9420 & -0.0158 & $0.057^{*}$ \\
\hline $\mathrm{H} 11 \mathrm{C}$ & 0.3361 & 0.9993 & 0.0358 & $0.057^{*}$ \\
\hline $\mathrm{C} 12 \mathrm{~A}$ & $0.7746(3)$ & $0.97312(15)$ & $0.17935(14)$ & $0.0375(8)$ \\
\hline $\mathrm{H} 12 \mathrm{~A}$ & 0.8564 & 0.9470 & 0.1856 & $0.056^{*}$ \\
\hline H12B & 0.7536 & 0.9824 & 0.2196 & $0.056^{*}$ \\
\hline $\mathrm{H} 12 \mathrm{C}$ & 0.7838 & 1.0174 & 0.1586 & $0.056^{*}$ \\
\hline N1B & $0.9217(2)$ & $0.79655(12)$ & $0.22174(11)$ & $0.0244(5)$ \\
\hline $\mathrm{H} 1 \mathrm{BB}$ & $0.837(3)$ & $0.8075(16)$ & $0.1961(14)$ & $0.050(9)^{*}$ \\
\hline $\mathrm{N} 2 \mathrm{~B}$ & $1.0990(2)$ & $0.72567(11)$ & $0.21211(10)$ & $0.0216(5)$ \\
\hline N3B & $0.9013(2)$ & $0.73288(11)$ & $0.13366(10)$ & $0.0232(5)$ \\
\hline C1B & $0.8653(3)$ & $0.85002(14)$ & $0.30962(13)$ & $0.0295(7)$ \\
\hline H1BA & 0.7786 & 0.8535 & 0.2858 & $0.035^{*}$ \\
\hline $\mathrm{C} 2 \mathrm{~B}$ & $0.8942(3)$ & $0.87347(14)$ & $0.36999(13)$ & $0.0316(7)$ \\
\hline $\mathrm{H} 2 \mathrm{BA}$ & 0.8275 & 0.8927 & 0.3876 & $0.038^{*}$ \\
\hline C3B & $1.0206(3)$ & $0.86906(14)$ & $0.40526(14)$ & $0.0326(7)$ \\
\hline H3BA & 1.0406 & 0.8848 & 0.4470 & $0.039^{*}$ \\
\hline
\end{tabular}




\begin{tabular}{|c|c|c|c|c|}
\hline C4B & $1.1167(3)$ & $0.84163(14)$ & $0.37901(13)$ & $0.0299(7)$ \\
\hline H4BA & 1.2033 & 0.8386 & 0.4030 & $0.036^{*}$ \\
\hline $\mathrm{C} 5 \mathrm{~B}$ & $1.0887(3)$ & $0.81842(14)$ & $0.31799(13)$ & $0.0261(7)$ \\
\hline H5BA & 1.1563 & 0.8007 & 0.3002 & $0.031 *$ \\
\hline C6B & $0.9619(2)$ & $0.82115(13)$ & $0.28306(12)$ & $0.0222(6)$ \\
\hline C7B & $0.9786(2)$ & $0.74942(14)$ & $0.18899(12)$ & $0.0220(6)$ \\
\hline $\mathrm{C} 8 \mathrm{~B}$ & $1.1475(2)$ & $0.68135(14)$ & $0.17509(12)$ & $0.0229(6)$ \\
\hline C9B & $1.0768(3)$ & $0.66375(14)$ & $0.11680(13)$ & $0.0247(7)$ \\
\hline H9BA & 1.1128 & 0.6339 & 0.0907 & $0.030^{*}$ \\
\hline $\mathrm{C} 10 \mathrm{~B}$ & $0.9522(3)$ & $0.69043(14)$ & $0.09717(12)$ & $0.0233(6)$ \\
\hline C11B & $1.2817(2)$ & $0.65336(15)$ & $0.20046(13)$ & $0.0304(7)$ \\
\hline H11D & 1.3404 & 0.6919 & 0.2169 & $0.046^{*}$ \\
\hline H11E & 1.3134 & 0.6298 & 0.1673 & $0.046^{*}$ \\
\hline $\mathrm{H} 11 \mathrm{~F}$ & 1.2791 & 0.6201 & 0.2338 & $0.046^{*}$ \\
\hline C12B & $0.8698(3)$ & $0.67444(15)$ & $0.03397(12)$ & $0.0307(7)$ \\
\hline H12D & 0.7828 & 0.6944 & 0.0303 & $0.046^{*}$ \\
\hline $\mathrm{H} 12 \mathrm{E}$ & 0.8627 & 0.6238 & 0.0283 & $0.046^{*}$ \\
\hline $\mathrm{H} 12 \mathrm{~F}$ & 0.9103 & 0.6947 & 0.0022 & $0.046^{*}$ \\
\hline $\mathrm{N} 1 \mathrm{C}$ & $1.1500(2)$ & $1.08100(12)$ & $0.13645(11)$ & $0.0274(6)$ \\
\hline $\mathrm{H} 1 \mathrm{CB}$ & $1.239(3)$ & $1.0913(16)$ & $0.1519(15)$ & $0.058(10)^{*}$ \\
\hline $\mathrm{N} 2 \mathrm{C}$ & $1.0238(2)$ & $0.98550(12)$ & $0.09055(10)$ & $0.0285(6)$ \\
\hline $\mathrm{N} 3 \mathrm{C}$ & $1.2273(2)$ & $0.97203(12)$ & $0.16432(10)$ & $0.0254(5)$ \\
\hline $\mathrm{C} 1 \mathrm{C}$ & $1.1158(3)$ & $1.20305(14)$ & $0.13836(12)$ & $0.0280(7)$ \\
\hline $\mathrm{H} 1 \mathrm{CA}$ & 1.2015 & 1.2066 & 0.1632 & $0.034 *$ \\
\hline $\mathrm{C} 2 \mathrm{C}$ & $1.0416(3)$ & $1.26280(15)$ & $0.12328(13)$ & $0.0333(7)$ \\
\hline $\mathrm{H} 2 \mathrm{CA}$ & 1.0768 & 1.3069 & 0.1377 & $0.040^{*}$ \\
\hline $\mathrm{C} 3 \mathrm{C}$ & $0.9166(3)$ & $1.25855(16)$ & 0.08734 (13) & $0.0360(8)$ \\
\hline $\mathrm{H} 3 \mathrm{CA}$ & 0.8647 & 1.2993 & 0.0776 & $0.043 *$ \\
\hline $\mathrm{C} 4 \mathrm{C}$ & $0.8683(3)$ & $1.19384(17)$ & $0.06585(13)$ & $0.0357(8)$ \\
\hline $\mathrm{H} 4 \mathrm{CA}$ & 0.7833 & 1.1907 & 0.0402 & $0.043^{*}$ \\
\hline $\mathrm{C} 5 \mathrm{C}$ & $0.9410(3)$ & $1.13327(16)$ & $0.08091(13)$ & $0.0314(7)$ \\
\hline $\mathrm{H} 5 \mathrm{CA}$ & 0.9056 & 1.0892 & 0.0664 & $0.038^{*}$ \\
\hline $\mathrm{C} 6 \mathrm{C}$ & $1.0665(3)$ & $1.13804(14)$ & $0.11756(12)$ & $0.0247(7)$ \\
\hline $\mathrm{C} 7 \mathrm{C}$ & $1.1299(3)$ & $1.01019(14)$ & $0.12912(13)$ & $0.0252(7)$ \\
\hline $\mathrm{C} 8 \mathrm{C}$ & $1.0168(3)$ & $0.91572(15)$ & $0.08390(13)$ & $0.0284(7)$ \\
\hline $\mathrm{C} 9 \mathrm{C}$ & $1.1147(3)$ & $0.87277(15)$ & $0.11533(13)$ & $0.0302(7)$ \\
\hline H9CA & 1.1106 & 0.8238 & 0.1089 & $0.036^{*}$ \\
\hline $\mathrm{C} 10 \mathrm{C}$ & $1.2192(3)$ & $0.90258(14)$ & $0.15653(13)$ & $0.0269(7)$ \\
\hline $\mathrm{C} 11 \mathrm{C}$ & $0.8976(3)$ & $0.88820(16)$ & $0.04020(13)$ & $0.0352(8)$ \\
\hline $\mathrm{H} 11 \mathrm{G}$ & 0.8728 & 0.9200 & 0.0048 & $0.053^{*}$ \\
\hline $\mathrm{H} 11 \mathrm{H}$ & 0.8261 & 0.8847 & 0.0618 & $0.053^{*}$ \\
\hline H11I & 0.9161 & 0.8419 & 0.0254 & $0.053 *$ \\
\hline $\mathrm{C} 12 \mathrm{C}$ & $1.3266(3)$ & $0.85994(15)$ & $0.19480(13)$ & $0.0332(7)$ \\
\hline $\mathrm{H} 12 \mathrm{G}$ & 1.4079 & 0.8867 & 0.2023 & $0.050^{*}$ \\
\hline $\mathrm{H} 12 \mathrm{H}$ & 1.3372 & 0.8168 & 0.1726 & $0.050 *$ \\
\hline H12I & 1.3049 & 0.8484 & 0.2345 & $0.050 *$ \\
\hline N1D & $1.4540(2)$ & $1.03911(12)$ & $0.24960(11)$ & $0.0242(5)$ \\
\hline H1DB & $1.378(3)$ & $1.0251(15)$ & $0.2234(13)$ & $0.039(9)^{*}$ \\
\hline
\end{tabular}




$\begin{array}{lllll}\text { N2D } & 1.6334(2) & 1.10956(11) & 0.24278(10) & 0.0240(5) \\ \text { N3D } & 1.4352(2) & 1.10768(11) & 0.16432(10) & 0.0227(5) \\ \text { C1D } & 1.3924(3) & 0.98318(14) & 0.33508(13) & 0.0276(7) \\ \text { H1DA } & 1.3072 & 0.9788 & 0.3099 & 0.033^{*} \\ \text { C2D } & 1.4181(3) & 0.95921(14) & 0.39511(13) & 0.0290(7) \\ \text { H2DA } & 1.3501 & 0.9391 & 0.4112 & 0.035^{*} \\ \text { C3D } & 1.5421(3) & 0.96405(14) & 0.43236(13) & 0.0304(7) \\ \text { H3DA } & 1.5596 & 0.9478 & 0.4739 & 0.036^{*} \\ \text { C4D } & 1.6398(3) & 0.99302(14) & 0.40792(13) & 0.0285(7) \\ \text { H4DA } & 1.7253 & 0.9961 & 0.4331 & 0.034^{*} \\ \text { C5D } & 1.6162(2) & 1.01761(14) & 0.34767(12) & 0.0252(7) \\ \text { H5DA } & 1.6850 & 1.0368 & 0.3315 & 0.030^{*} \\ \text { C6D } & 1.4905(3) & 1.01402(13) & 0.31083(12) & 0.0227(6) \\ \text { C7D } & 1.5119(2) & 1.08765(13) & 0.21896(12) & 0.0211(6) \\ \text { C8D } & 1.6839(3) & 1.15457(14) & 0.20693(13) & 0.0259(7) \\ \text { C9D } & 1.6144(3) & 1.17560(14) & 0.14969(13) & 0.0264(7) \\ \text { H9DA } & 1.6521 & 1.2059 & 0.1246 & 0.032^{*} \\ \text { C10D } & 1.4874(3) & 1.15146(13) & 0.12938(12) & 0.0227(6) \\ \text { C11D } & 1.8201(3) & 1.17911(16) & 0.23313(14) & 0.0356(8) \\ \text { H11J } & 1.8746 & 1.1393 & 0.2503 & 0.053^{*} \\ \text { H11K } & 1.8195 & 1.2133 & 0.2660 & 0.053^{*} \\ \text { H11L } & 1.8553 & 1.2008 & 0.2002 & 0.053^{*} \\ \text { C12D } & 1.4050(3) & 1.17113(14) & 0.06759(12) & 0.0280(7) \\ \text { H12J } & 1.3215 & 1.1895 & 0.0731 & 0.042^{*} \\ \text { H12K } & 1.3897 & 1.1299 & 0.0408 & 0.042^{*} \\ \text { H12L } & 1.4499 & 1.2069 & 0.0484 & 0.042^{*} \\ & & & & \end{array}$

Atomic displacement parameters $\left(\AA^{2}\right)$

\begin{tabular}{lllllll}
\hline & $U^{11}$ & $U^{22}$ & $U^{33}$ & $U^{12}$ & $U^{13}$ & $U^{23}$ \\
\hline $\mathrm{N} 1 \mathrm{~A}$ & $0.0219(14)$ & $0.0189(13)$ & $0.0347(15)$ & $0.0032(11)$ & $-0.0007(12)$ & $-0.0004(11)$ \\
$\mathrm{N} 2 \mathrm{~A}$ & $0.0253(13)$ & $0.0271(14)$ & $0.0271(14)$ & $0.0056(10)$ & $0.0085(12)$ & $0.0052(11)$ \\
$\mathrm{N} 3 \mathrm{~A}$ & $0.0276(13)$ & $0.0204(13)$ & $0.0298(14)$ & $0.0016(10)$ & $0.0081(12)$ & $-0.0037(11)$ \\
$\mathrm{C} 1 \mathrm{~A}$ & $0.0240(15)$ & $0.0266(16)$ & $0.0311(17)$ & $0.0024(13)$ & $0.0011(14)$ & $0.0006(14)$ \\
$\mathrm{C} 2 \mathrm{~A}$ & $0.0337(18)$ & $0.0251(16)$ & $0.0398(19)$ & $0.0014(14)$ & $0.0085(16)$ & $-0.0032(14)$ \\
$\mathrm{C} 3 \mathrm{~A}$ & $0.0364(18)$ & $0.0294(17)$ & $0.0262(17)$ & $-0.0101(14)$ & $0.0059(15)$ & $-0.0022(14)$ \\
$\mathrm{C} 4 \mathrm{~A}$ & $0.0252(16)$ & $0.0427(19)$ & $0.0214(16)$ & $-0.0045(14)$ & $0.0009(14)$ & $0.0023(14)$ \\
$\mathrm{C} 5 \mathrm{~A}$ & $0.0265(16)$ & $0.0295(17)$ & $0.0251(17)$ & $0.0034(13)$ & $0.0021(14)$ & $0.0050(13)$ \\
$\mathrm{C}$ A & $0.0224(15)$ & $0.0254(16)$ & $0.0221(16)$ & $0.0013(12)$ & $0.0041(13)$ & $-0.0002(13)$ \\
$\mathrm{C} 7 \mathrm{~A}$ & $0.0283(16)$ & $0.0245(16)$ & $0.0232(17)$ & $0.0037(13)$ & $0.0110(14)$ & $0.0022(13)$ \\
$\mathrm{C} 8 \mathrm{~A}$ & $0.0303(16)$ & $0.0313(18)$ & $0.0291(18)$ & $0.0097(14)$ & $0.0152(15)$ & $0.0083(14)$ \\
$\mathrm{C} 9 \mathrm{~A}$ & $0.0382(18)$ & $0.0221(16)$ & $0.0357(19)$ & $0.0080(14)$ & $0.0150(16)$ & $0.0023(14)$ \\
$\mathrm{C} 10 \mathrm{~A}$ & $0.0340(16)$ & $0.0251(16)$ & $0.0310(18)$ & $0.0019(14)$ & $0.0164(15)$ & $-0.0032(14)$ \\
$\mathrm{C} 11 \mathrm{~A}$ & $0.0343(17)$ & $0.0355(18)$ & $0.044(2)$ & $0.0104(14)$ & $0.0087(16)$ & $0.0124(16)$ \\
$\mathrm{C} 12 \mathrm{~A}$ & $0.0437(19)$ & $0.0260(17)$ & $0.043(2)$ & $-0.0022(14)$ & $0.0105(17)$ & $-0.0082(15)$ \\
$\mathrm{N} 1 \mathrm{~B}$ & $0.0214(13)$ & $0.0265(13)$ & $0.0235(14)$ & $0.0034(11)$ & $0.0011(12)$ & $-0.0061(11)$ \\
$\mathrm{N} 2 \mathrm{~B}$ & $0.0212(12)$ & $0.0215(12)$ & $0.0229(13)$ & $0.0011(10)$ & $0.0062(11)$ & $0.0006(10)$ \\
$\mathrm{N} 3 \mathrm{~B}$ & $0.0262(12)$ & $0.0225(13)$ & $0.0203(13)$ & $-0.0026(10)$ & $0.0038(11)$ & $-0.0029(11)$
\end{tabular}




\begin{tabular}{|c|c|c|c|c|c|c|}
\hline $\mathrm{C} 1 \mathrm{~B}$ & $0.0302(16)$ & $0.0261(16)$ & $0.0338(18)$ & $0.0027(13)$ & $0.0105(15)$ & $-0.0036(14)$ \\
\hline $\mathrm{C} 2 \mathrm{~B}$ & $0.0387(18)$ & $0.0273(17)$ & $0.0317(19)$ & $0.0012(14)$ & $0.0138(16)$ & $-0.0061(14)$ \\
\hline $\mathrm{C} 3 \mathrm{~B}$ & $0.046(2)$ & $0.0250(16)$ & $0.0273(17)$ & $-0.0012(14)$ & $0.0084(16)$ & $-0.0056(14)$ \\
\hline C4B & $0.0303(16)$ & $0.0299(17)$ & $0.0280(18)$ & $-0.0013(13)$ & $0.0034(14)$ & $-0.0032(14)$ \\
\hline $\mathrm{C} 5 \mathrm{~B}$ & $0.0274(16)$ & $0.0236(15)$ & $0.0281(17)$ & $0.0008(13)$ & $0.0081(14)$ & -0.0024 \\
\hline $\mathrm{C} 6 \mathrm{~B}$ & $0.0251(16)$ & $0.0171(14)$ & $0.0234(16)$ & $-0.0022(12)$ & $0.0030(14)$ & $-0.0005(12)$ \\
\hline $\mathrm{C} 7 \mathrm{~B}$ & $0.0221(15)$ & $0.0205(14)$ & $0.0240(16)$ & $-0.0028(12)$ & $0.0062(14)$ & 0.0005 \\
\hline $\mathrm{C} 8 \mathrm{~B}$ & $0.0239(15)$ & $0.0225(15)$ & $0.0233(16)$ & $0.0022(12)$ & $0.0076(14)$ & $0.0053(13)$ \\
\hline C9B & $0.0284(16)$ & $0.0231(15)$ & $0.0250(17)$ & $0.0006(12)$ & $0.0109(14)$ & -0.0038 \\
\hline C10B & $0.0288(15)$ & $0.0207(15)$ & $0.0212(16)$ & $-0.0027(12)$ & $0.0073(14)$ & $0.0024(13)$ \\
\hline C11B & $0.0278(16)$ & $0.0327(17)$ & $0.0294(17)$ & $0.0072(13)$ & $0.0032(14)$ & $-0.0010(14)$ \\
\hline C12B & $0.0320(16)$ & $0.0324(17)$ & $0.0267(17)$ & $0.0003(14)$ & $0.0043(14)$ & $-0.0022(14)$ \\
\hline $\mathrm{N} 1 \mathrm{C}$ & $0.0240(14)$ & $0.0253(14)$ & $0.0301(15)$ & $-0.0001(11)$ & $-0.0002(12)$ & $-0.0008(11)$ \\
\hline $\mathrm{N} 2 \mathrm{C}$ & $0.0263(13)$ & $0.0323(14)$ & $0.0247(14)$ & $-0.0054(11)$ & $0.0005(12)$ & $0.0002(11)$ \\
\hline $\mathrm{N} 3 \mathrm{C}$ & $0.0243(13)$ & $0.0256(14)$ & $0.0248(14)$ & $-0.0040(10)$ & $0.0022(11)$ & $0.0030(11)$ \\
\hline $\mathrm{C} 1 \mathrm{C}$ & $0.0257(15)$ & $0.0320(17)$ & $0.0253(17)$ & $-0.0002(13)$ & $0.0035(14)$ & $0.0034(14)$ \\
\hline $\mathrm{C} 2 \mathrm{C}$ & $0.0367(18)$ & $0.0299(17)$ & $0.0337(18)$ & $0.0038(14)$ & $0.0086(16)$ & $0.0043(15)$ \\
\hline $\mathrm{C} 3 \mathrm{C}$ & 0.0395 (19) & $0.0359(19)$ & $0.0348(19)$ & $0.0111(15)$ & $0.0130(17)$ & $0.0109(15)$ \\
\hline $\mathrm{C} 4 \mathrm{C}$ & $0.0289(17)$ & $0.051(2)$ & $0.0263(18)$ & $0.0053(15)$ & $0.0037(15)$ & $0.0039(16)$ \\
\hline $\mathrm{C} 5 \mathrm{C}$ & $0.0263(16)$ & $0.0366(18)$ & $0.0310(18)$ & $0.0012(14)$ & $0.0057(15)$ & $-0.0008(14)$ \\
\hline $\mathrm{C} 6 \mathrm{C}$ & $0.0254(16)$ & $0.0293(17)$ & $0.0195(16)$ & $0.0028(13)$ & $0.0055(14)$ & $0.0032(13)$ \\
\hline $\mathrm{C} 7 \mathrm{C}$ & $0.0232(16)$ & $0.0297(17)$ & $0.0218(16)$ & $-0.0043(13)$ & $0.0030(14)$ & $0.0020(13)$ \\
\hline $\mathrm{C} 8 \mathrm{C}$ & $0.0285(16)$ & $0.0322(18)$ & $0.0250(17)$ & $-0.0099(14)$ & $0.0071(14)$ & $0.0019(14)$ \\
\hline $\mathrm{C} 9 \mathrm{C}$ & $0.0362(17)$ & $0.0253(16)$ & $0.0283(17)$ & $-0.0101(14)$ & $0.0056(15)$ & $0.0017(14)$ \\
\hline $\mathrm{C} 10 \mathrm{C}$ & $0.0293(16)$ & $0.0268(17)$ & $0.0254(17)$ & $-0.0035(13)$ & 0.0079 (14) & $0.0033(13)$ \\
\hline $\mathrm{C} 11 \mathrm{C}$ & $0.0334(17)$ & $0.0402(19)$ & $0.0289(17)$ & $-0.0138(14)$ & $0.0003(15)$ & $-0.0041(15)$ \\
\hline $\mathrm{C} 12 \mathrm{C}$ & 0.0367 (17) & $0.0281(17)$ & $0.0327(18)$ & $-0.0041(14)$ & $0.0033(15)$ & $0.0062(14)$ \\
\hline N1D & $0.0219(13)$ & $0.0243(13)$ & $0.0228(14)$ & $-0.0046(11)$ & $-0.0032(12)$ & $0.0054(11)$ \\
\hline $\mathrm{N} 2 \mathrm{D}$ & $0.0226(13)$ & $0.0231(13)$ & $0.0264(13)$ & $-0.0004(10)$ & 0.0059 (11) & $-0.0020(11)$ \\
\hline N3D & $0.0238(12)$ & $0.0205(12)$ & $0.0235(13)$ & $0.0034(10)$ & $0.0048(11)$ & $0.0039(11)$ \\
\hline C1D & $0.0222(15)$ & $0.0250(16)$ & $0.0329(18)$ & $-0.0002(12)$ & $0.0006(14)$ & $0.0049(14)$ \\
\hline $\mathrm{C} 2 \mathrm{D}$ & $0.0318(17)$ & $0.0255(16)$ & $0.0310(18)$ & $-0.0005(13)$ & $0.0095(15)$ & $0.0070(14)$ \\
\hline C3D & 0.0424 (19) & $0.0229(16)$ & $0.0258(17)$ & $0.0080(14)$ & $0.0076(16)$ & $0.0027(13)$ \\
\hline C4D & $0.0258(16)$ & $0.0296(17)$ & $0.0278(17)$ & $0.0061(13)$ & 0.0007 (14) & $-0.0003(14)$ \\
\hline C5D & $0.0201(15)$ & $0.0281(16)$ & $0.0256(17)$ & $-0.0009(12)$ & $0.0012(14)$ & $-0.0007(13)$ \\
\hline C6D & $0.0303(16)$ & $0.0163(14)$ & $0.0211(16)$ & $0.0021(12)$ & $0.0052(14)$ & $-0.0001(12)$ \\
\hline C7D & $0.0229(15)$ & $0.0171(14)$ & $0.0238(16)$ & $0.0021(12)$ & $0.0059(14)$ & $-0.0031(13)$ \\
\hline $\mathrm{C} 8 \mathrm{D}$ & $0.0270(15)$ & $0.0265(16)$ & $0.0254(17)$ & $-0.0021(13)$ & $0.0083(14)$ & $-0.0026(14)$ \\
\hline C9D & $0.0288(16)$ & $0.0274(16)$ & $0.0259(17)$ & $-0.0020(13)$ & $0.0119(15)$ & $0.0000(13)$ \\
\hline C10D & $0.0289(16)$ & $0.0177(14)$ & $0.0222(16)$ & $0.0043(12)$ & $0.0072(14)$ & $-0.0033(12)$ \\
\hline C11D & $0.0291(16)$ & $0.0438(19)$ & $0.0348(19)$ & $-0.0069(14)$ & $0.0089(15)$ & $0.0015(15)$ \\
\hline C12D & $0.0337(16)$ & $0.0231(16)$ & $0.0269(17)$ & $0.0004(13)$ & $0.0059(14)$ & $0.0012(13)$ \\
\hline
\end{tabular}

Geometric parameters $\left(\AA,{ }^{\circ}\right)$

\begin{tabular}{llll}
\hline N1A-C7A & $1.368(3)$ & $\mathrm{N} 1 \mathrm{C}-\mathrm{C} 7 \mathrm{C}$ & $1.378(3)$ \\
$\mathrm{N} 1 \mathrm{~A}-\mathrm{C} 6 \mathrm{~A}$ & $1.407(3)$ & $\mathrm{N} 1 \mathrm{C}-\mathrm{C} 6 \mathrm{C}$ & $1.408(3)$ \\
$\mathrm{N} 1 \mathrm{~A}-\mathrm{H} 1 \mathrm{AB}$ & $0.91(3)$ & $\mathrm{N} 1 \mathrm{C}-\mathrm{H} 1 \mathrm{CB}$ & $0.94(3)$
\end{tabular}




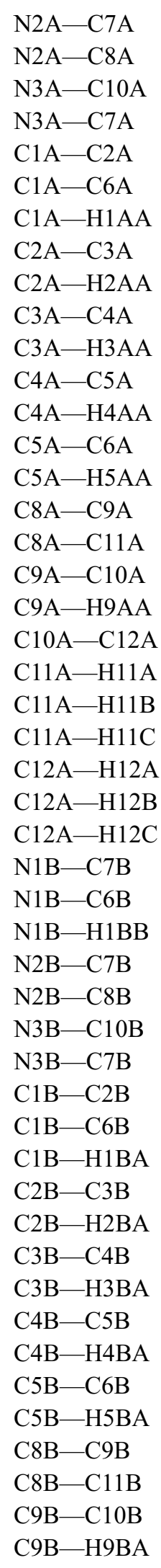

$1.338(3)$

$1.353(3)$

$1.340(3)$

$1.354(3)$

$1.380(4)$

$1.398(4)$

0.9500

$1.390(4)$

0.9500

1.387 (4)

0.9500

$1.383(4)$

0.9500

$1.390(4)$

0.9500

$1.380(4)$

$1.501(4)$

$1.385(4)$

0.9500

$1.504(4)$

0.9800

0.9800

0.9800

0.9800

0.9800

0.9800

$1.375(3)$

$1.410(3)$

0.97 (3)

$1.338(3)$

$1.356(3)$

$1.339(3)$

$1.349(3)$

$1.378(4)$

$1.397(3)$

0.9500

1.389 (4)

0.9500

$1.378(4)$

0.9500

1.389 (4)

0.9500

$1.388(4)$

0.9500

$1.381(4)$

$1.500(4)$

$1.386(4)$

0.9500
$\mathrm{N} 2 \mathrm{C}-\mathrm{C} 7 \mathrm{C}$

$\mathrm{N} 2 \mathrm{C}-\mathrm{C} 8 \mathrm{C}$

$\mathrm{N} 3 \mathrm{C}-\mathrm{C} 10 \mathrm{C}$

$\mathrm{N} 3 \mathrm{C}-\mathrm{C} 7 \mathrm{C}$

$\mathrm{C} 1 \mathrm{C}-\mathrm{C} 2 \mathrm{C}$

$\mathrm{C} 1 \mathrm{C}-\mathrm{C} 6 \mathrm{C}$

C1C-H1CA

$\mathrm{C} 2 \mathrm{C}-\mathrm{C} 3 \mathrm{C}$

$\mathrm{C} 2 \mathrm{C}-\mathrm{H} 2 \mathrm{CA}$

$\mathrm{C} 3 \mathrm{C}-\mathrm{C} 4 \mathrm{C}$

$\mathrm{C} 3 \mathrm{C}-\mathrm{H} 3 \mathrm{CA}$

$\mathrm{C} 4 \mathrm{C}-\mathrm{C} 5 \mathrm{C}$

$\mathrm{C} 4 \mathrm{C}-\mathrm{H} 4 \mathrm{CA}$

$\mathrm{C} 5 \mathrm{C}-\mathrm{C} 6 \mathrm{C}$

$\mathrm{C} 5 \mathrm{C}-\mathrm{H} 5 \mathrm{CA}$

$\mathrm{C} 8 \mathrm{C}-\mathrm{C} 9 \mathrm{C}$

$\mathrm{C} 8 \mathrm{C}-\mathrm{C} 11 \mathrm{C}$

$\mathrm{C} 9 \mathrm{C}-\mathrm{C} 10 \mathrm{C}$

C9C-H9CA

C10C-C12C

$\mathrm{C} 11 \mathrm{C}-\mathrm{H} 11 \mathrm{G}$

C11C- $-\mathrm{H} 11 \mathrm{H}$

C11C-H11I

$\mathrm{C} 12 \mathrm{C}-\mathrm{H} 12 \mathrm{G}$

$\mathrm{C} 12 \mathrm{C}-\mathrm{H} 12 \mathrm{H}$

$\mathrm{C} 12 \mathrm{C}-\mathrm{H} 12 \mathrm{I}$

N1D - C7D

N1D-C6D

N1D-H1DB

N2D - C7D

$\mathrm{N} 2 \mathrm{D}-\mathrm{C} 8 \mathrm{D}$

N3D-C10D

N3D $-C 7 D$

C1D $-C 2 D$

C1D-C6D

C1D-H1DA

C2D-C3D

C2D-H2DA

C3D-C4D

C3D-H3DA

C4D-C5D

C4D-H4DA

C5D-C6D

C5D-H5DA

C8D-C9D

C8D-C11D

C9D-C10D

C9D-H9DA
$1.334(3)$

$1.346(3)$

$1.343(3)$

1.357 (3)

1.385 (4)

$1.389(4)$

0.9500

$1.382(4)$

0.9500

1.384 (4)

0.9500

$1.391(4)$

0.9500

$1.392(4)$

0.9500

$1.382(4)$

$1.501(4)$

1.387 (4)

0.9500

1.497 (4)

0.9800

0.9800

0.9800

0.9800

0.9800

0.9800

$1.371(3)$

1.408 (3)

0.92 (3)

$1.339(3)$

1.357 (3)

$1.339(3)$

$1.352(3)$

$1.374(4)$

1.397 (3)

0.9500

1.384 (4)

0.9500

1.380 (4)

0.9500

$1.383(4)$

0.9500

1.394 (4)

0.9500

$1.375(4)$

$1.500(4)$

$1.392(4)$

0.9500 


\begin{tabular}{|c|c|c|c|}
\hline $\mathrm{C} 10 \mathrm{~B}-\mathrm{C} 12 \mathrm{~B}$ & $1.505(4)$ & $\mathrm{C} 10 \mathrm{D}-\mathrm{C} 12 \mathrm{D}$ & $1.496(4)$ \\
\hline C11B-H11D & 0.9800 & $\mathrm{C} 11 \mathrm{D}-\mathrm{H} 11 \mathrm{~J}$ & 0.9800 \\
\hline $\mathrm{C} 11 \mathrm{~B}-\mathrm{H} 11 \mathrm{E}$ & 0.9800 & $\mathrm{C} 11 \mathrm{D}-\mathrm{H} 11 \mathrm{~K}$ & 0.9800 \\
\hline $\mathrm{C} 11 \mathrm{~B}-\mathrm{H} 11 \mathrm{~F}$ & 0.9800 & C11D-H11L & 0.9800 \\
\hline $\mathrm{C} 12 \mathrm{~B}-\mathrm{H} 12 \mathrm{D}$ & 0.9800 & $\mathrm{C} 12 \mathrm{D}-\mathrm{H} 12 \mathrm{~J}$ & 0.9800 \\
\hline $\mathrm{C} 12 \mathrm{~B}-\mathrm{H} 12 \mathrm{E}$ & 0.9800 & $\mathrm{C} 12 \mathrm{D}-\mathrm{H} 12 \mathrm{~K}$ & 0.9800 \\
\hline $\mathrm{C} 12 \mathrm{~B}-\mathrm{H} 12 \mathrm{~F}$ & 0.9800 & $\mathrm{C} 12 \mathrm{D}-\mathrm{H} 12 \mathrm{~L}$ & 0.9800 \\
\hline $\mathrm{C} 7 \mathrm{~A}-\mathrm{N} 1 \mathrm{~A}-\mathrm{C} 6 \mathrm{~A}$ & $131.9(2)$ & $\mathrm{C} 7 \mathrm{C}-\mathrm{N} 1 \mathrm{C}-\mathrm{C} 6 \mathrm{C}$ & $131.4(3)$ \\
\hline $\mathrm{C} 7 \mathrm{~A}-\mathrm{N} 1 \mathrm{~A}-\mathrm{H} 1 \mathrm{AB}$ & $111.3(17)$ & $\mathrm{C} 7 \mathrm{C}-\mathrm{N} 1 \mathrm{C}-\mathrm{H} 1 \mathrm{CB}$ & $111(2)$ \\
\hline $\mathrm{C} 6 \mathrm{~A}-\mathrm{N} 1 \mathrm{~A}-\mathrm{H} 1 \mathrm{AB}$ & $116.8(16)$ & $\mathrm{C} 6 \mathrm{C}-\mathrm{N} 1 \mathrm{C}-\mathrm{H} 1 \mathrm{CB}$ & $117(2)$ \\
\hline $\mathrm{C} 7 \mathrm{~A}-\mathrm{N} 2 \mathrm{~A}-\mathrm{C} 8 \mathrm{~A}$ & $115.7(3)$ & $\mathrm{C} 7 \mathrm{C}-\mathrm{N} 2 \mathrm{C}-\mathrm{C} 8 \mathrm{C}$ & $116.1(3)$ \\
\hline $\mathrm{C} 10 \mathrm{~A}-\mathrm{N} 3 \mathrm{~A}-\mathrm{C} 7 \mathrm{~A}$ & $116.2(3)$ & $\mathrm{C} 10 \mathrm{C}-\mathrm{N} 3 \mathrm{C}-\mathrm{C} 7 \mathrm{C}$ & $116.3(2)$ \\
\hline $\mathrm{C} 2 \mathrm{~A}-\mathrm{C} 1 \mathrm{~A}-\mathrm{C} 6 \mathrm{~A}$ & $121.0(3)$ & $\mathrm{C} 2 \mathrm{C}-\mathrm{C} 1 \mathrm{C}-\mathrm{C} 6 \mathrm{C}$ & $120.8(3)$ \\
\hline $\mathrm{C} 2 \mathrm{~A}-\mathrm{C} 1 \mathrm{~A}-\mathrm{H} 1 \mathrm{AA}$ & 119.5 & $\mathrm{C} 2 \mathrm{C}-\mathrm{C} 1 \mathrm{C}-\mathrm{H} 1 \mathrm{CA}$ & 119.6 \\
\hline $\mathrm{C} 6 \mathrm{~A}-\mathrm{C} 1 \mathrm{~A}-\mathrm{H} 1 \mathrm{AA}$ & 119.5 & $\mathrm{C} 6 \mathrm{C}-\mathrm{C} 1 \mathrm{C}-\mathrm{H} 1 \mathrm{CA}$ & 119.6 \\
\hline $\mathrm{C} 1 \mathrm{~A}-\mathrm{C} 2 \mathrm{~A}-\mathrm{C} 3 \mathrm{~A}$ & $120.3(3)$ & $\mathrm{C} 3 \mathrm{C}-\mathrm{C} 2 \mathrm{C}-\mathrm{C} 1 \mathrm{C}$ & $120.3(3)$ \\
\hline $\mathrm{C} 1 \mathrm{~A}-\mathrm{C} 2 \mathrm{~A}-\mathrm{H} 2 \mathrm{AA}$ & 119.8 & $\mathrm{C} 3 \mathrm{C}-\mathrm{C} 2 \mathrm{C}-\mathrm{H} 2 \mathrm{CA}$ & 119.8 \\
\hline $\mathrm{C} 3 \mathrm{~A}-\mathrm{C} 2 \mathrm{~A}-\mathrm{H} 2 \mathrm{AA}$ & 119.8 & $\mathrm{C} 1 \mathrm{C}-\mathrm{C} 2 \mathrm{C}-\mathrm{H} 2 \mathrm{CA}$ & 119.8 \\
\hline $\mathrm{C} 4 \mathrm{~A}-\mathrm{C} 3 \mathrm{~A}-\mathrm{C} 2 \mathrm{~A}$ & $118.6(3)$ & $\mathrm{C} 2 \mathrm{C}-\mathrm{C} 3 \mathrm{C}-\mathrm{C} 4 \mathrm{C}$ & $118.8(3)$ \\
\hline $\mathrm{C} 4 \mathrm{~A}-\mathrm{C} 3 \mathrm{~A}-\mathrm{H} 3 \mathrm{AA}$ & 120.7 & $\mathrm{C} 2 \mathrm{C}-\mathrm{C} 3 \mathrm{C}-\mathrm{H} 3 \mathrm{CA}$ & 120.6 \\
\hline $\mathrm{C} 2 \mathrm{~A}-\mathrm{C} 3 \mathrm{~A}-\mathrm{H} 3 \mathrm{AA}$ & 120.7 & $\mathrm{C} 4 \mathrm{C}-\mathrm{C} 3 \mathrm{C}-\mathrm{H} 3 \mathrm{CA}$ & 120.6 \\
\hline $\mathrm{C} 5 \mathrm{~A}-\mathrm{C} 4 \mathrm{~A}-\mathrm{C} 3 \mathrm{~A}$ & $121.6(3)$ & $\mathrm{C} 3 \mathrm{C}-\mathrm{C} 4 \mathrm{C}-\mathrm{C} 5 \mathrm{C}$ & $121.6(3)$ \\
\hline $\mathrm{C} 5 \mathrm{~A}-\mathrm{C} 4 \mathrm{~A}-\mathrm{H} 4 \mathrm{AA}$ & 119.2 & $\mathrm{C} 3 \mathrm{C}-\mathrm{C} 4 \mathrm{C}-\mathrm{H} 4 \mathrm{CA}$ & 119.2 \\
\hline $\mathrm{C} 3 \mathrm{~A}-\mathrm{C} 4 \mathrm{~A}-\mathrm{H} 4 \mathrm{AA}$ & 119.2 & $\mathrm{C} 5 \mathrm{C}-\mathrm{C} 4 \mathrm{C}-\mathrm{H} 4 \mathrm{CA}$ & 119.2 \\
\hline $\mathrm{C} 4 \mathrm{~A}-\mathrm{C} 5 \mathrm{~A}-\mathrm{C} 6 \mathrm{~A}$ & $119.8(3)$ & $\mathrm{C} 4 \mathrm{C}-\mathrm{C} 5 \mathrm{C}-\mathrm{C} 6 \mathrm{C}$ & $119.1(3)$ \\
\hline $\mathrm{C} 4 \mathrm{~A}-\mathrm{C} 5 \mathrm{~A}-\mathrm{H} 5 \mathrm{AA}$ & 120.1 & $\mathrm{C} 4 \mathrm{C}-\mathrm{C} 5 \mathrm{C}-\mathrm{H} 5 \mathrm{CA}$ & 120.4 \\
\hline $\mathrm{C} 6 \mathrm{~A}-\mathrm{C} 5 \mathrm{~A}-\mathrm{H} 5 \mathrm{AA}$ & 120.1 & $\mathrm{C} 6 \mathrm{C}-\mathrm{C} 5 \mathrm{C}-\mathrm{H} 5 \mathrm{CA}$ & 120.4 \\
\hline $\mathrm{C} 5 \mathrm{~A}-\mathrm{C} 6 \mathrm{~A}-\mathrm{C} 1 \mathrm{~A}$ & $118.7(3)$ & $\mathrm{C} 1 \mathrm{C}-\mathrm{C} 6 \mathrm{C}-\mathrm{C} 5 \mathrm{C}$ & $119.3(3)$ \\
\hline $\mathrm{C} 5 \mathrm{~A}-\mathrm{C} 6 \mathrm{~A}-\mathrm{N} 1 \mathrm{~A}$ & $125.4(3)$ & $\mathrm{C} 1 \mathrm{C}-\mathrm{C} 6 \mathrm{C}-\mathrm{N} 1 \mathrm{C}$ & $115.8(2)$ \\
\hline $\mathrm{C} 1 \mathrm{~A}-\mathrm{C} 6 \mathrm{~A}-\mathrm{N} 1 \mathrm{~A}$ & $115.8(2)$ & $\mathrm{C} 5 \mathrm{C}-\mathrm{C} 6 \mathrm{C}-\mathrm{N} 1 \mathrm{C}$ & $124.9(3)$ \\
\hline $\mathrm{N} 2 \mathrm{~A}-\mathrm{C} 7 \mathrm{~A}-\mathrm{N} 3 \mathrm{~A}$ & $126.8(2)$ & $\mathrm{N} 2 \mathrm{C}-\mathrm{C} 7 \mathrm{C}-\mathrm{N} 3 \mathrm{C}$ & $126.6(3)$ \\
\hline $\mathrm{N} 2 \mathrm{~A}-\mathrm{C} 7 \mathrm{~A}-\mathrm{N} 1 \mathrm{~A}$ & $120.6(3)$ & $\mathrm{N} 2 \mathrm{C}-\mathrm{C} 7 \mathrm{C}-\mathrm{N} 1 \mathrm{C}$ & $120.7(3)$ \\
\hline $\mathrm{N} 3 \mathrm{~A}-\mathrm{C} 7 \mathrm{~A}-\mathrm{N} 1 \mathrm{~A}$ & $112.5(2)$ & $\mathrm{N} 3 \mathrm{C}-\mathrm{C} 7 \mathrm{C}-\mathrm{N} 1 \mathrm{C}$ & $112.7(2)$ \\
\hline $\mathrm{N} 2 \mathrm{~A}-\mathrm{C} 8 \mathrm{~A}-\mathrm{C} 9 \mathrm{~A}$ & $121.5(3)$ & $\mathrm{N} 2 \mathrm{C}-\mathrm{C} 8 \mathrm{C}-\mathrm{C} 9 \mathrm{C}$ & $121.3(3)$ \\
\hline $\mathrm{N} 2 \mathrm{~A}-\mathrm{C} 8 \mathrm{~A}-\mathrm{C} 11 \mathrm{~A}$ & $116.2(3)$ & $\mathrm{N} 2 \mathrm{C}-\mathrm{C} 8 \mathrm{C}-\mathrm{C} 11 \mathrm{C}$ & $116.0(3)$ \\
\hline $\mathrm{C} 9 \mathrm{~A}-\mathrm{C} 8 \mathrm{~A}-\mathrm{C} 11 \mathrm{~A}$ & $122.2(3)$ & $\mathrm{C} 9 \mathrm{C}-\mathrm{C} 8 \mathrm{C}-\mathrm{C} 11 \mathrm{C}$ & $122.7(3)$ \\
\hline $\mathrm{C} 8 \mathrm{~A}-\mathrm{C} 9 \mathrm{~A}-\mathrm{C} 10 \mathrm{~A}$ & $118.6(3)$ & $\mathrm{C} 8 \mathrm{C}-\mathrm{C} 9 \mathrm{C}-\mathrm{C} 10 \mathrm{C}$ & $118.7(3)$ \\
\hline $\mathrm{C} 8 \mathrm{~A}-\mathrm{C} 9 \mathrm{~A}-\mathrm{H} 9 \mathrm{AA}$ & 120.7 & $\mathrm{C} 8 \mathrm{C}-\mathrm{C} 9 \mathrm{C}-\mathrm{H} 9 \mathrm{CA}$ & 120.6 \\
\hline $\mathrm{C} 10 \mathrm{~A}-\mathrm{C} 9 \mathrm{~A}-\mathrm{H} 9 \mathrm{AA}$ & 120.7 & $\mathrm{C} 10 \mathrm{C}-\mathrm{C} 9 \mathrm{C}-\mathrm{H} 9 \mathrm{CA}$ & 120.6 \\
\hline $\mathrm{N} 3 \mathrm{~A}-\mathrm{C} 10 \mathrm{~A}-\mathrm{C} 9 \mathrm{~A}$ & $121.1(3)$ & $\mathrm{N} 3 \mathrm{C}-\mathrm{C} 10 \mathrm{C}-\mathrm{C} 9 \mathrm{C}$ & $120.8(3)$ \\
\hline $\mathrm{N} 3 \mathrm{~A}-\mathrm{C} 10 \mathrm{~A}-\mathrm{C} 12 \mathrm{~A}$ & $117.0(3)$ & $\mathrm{N} 3 \mathrm{C}-\mathrm{C} 10 \mathrm{C}-\mathrm{C} 12 \mathrm{C}$ & $116.8(3)$ \\
\hline $\mathrm{C} 9 \mathrm{~A}-\mathrm{C} 10 \mathrm{~A}-\mathrm{C} 12 \mathrm{~A}$ & $121.9(3)$ & $\mathrm{C} 9 \mathrm{C}-\mathrm{C} 10 \mathrm{C}-\mathrm{C} 12 \mathrm{C}$ & $122.5(3)$ \\
\hline $\mathrm{C} 8 \mathrm{~A}-\mathrm{C} 11 \mathrm{~A}-\mathrm{H} 11 \mathrm{~A}$ & 109.5 & $\mathrm{C} 8 \mathrm{C}-\mathrm{C} 11 \mathrm{C}-\mathrm{H} 11 \mathrm{G}$ & 109.5 \\
\hline $\mathrm{C} 8 \mathrm{~A}-\mathrm{C} 11 \mathrm{~A}-\mathrm{H} 11 \mathrm{~B}$ & 109.5 & $\mathrm{C} 8 \mathrm{C}-\mathrm{C} 11 \mathrm{C}-\mathrm{H} 11 \mathrm{H}$ & 109.5 \\
\hline $\mathrm{H} 11 \mathrm{~A}-\mathrm{C} 11 \mathrm{~A}-\mathrm{H} 11 \mathrm{~B}$ & 109.5 & $\mathrm{H} 11 \mathrm{G}-\mathrm{C} 11 \mathrm{C}-\mathrm{H} 11 \mathrm{H}$ & 109.5 \\
\hline $\mathrm{C} 8 \mathrm{~A}-\mathrm{C} 11 \mathrm{~A}-\mathrm{H} 11 \mathrm{C}$ & 109.5 & $\mathrm{C} 8 \mathrm{C}-\mathrm{C} 11 \mathrm{C}-\mathrm{H} 11 \mathrm{I}$ & 109.5 \\
\hline $\mathrm{H} 11 \mathrm{~A}-\mathrm{C} 11 \mathrm{~A}-\mathrm{H} 11 \mathrm{C}$ & 109.5 & $\mathrm{H} 11 \mathrm{G}-\mathrm{C} 11 \mathrm{C}-\mathrm{H} 11 \mathrm{I}$ & 109.5 \\
\hline
\end{tabular}




\begin{tabular}{|c|c|c|c|}
\hline $\mathrm{H} 11 \mathrm{~B}-\mathrm{C} 11 \mathrm{~A}-\mathrm{H} 11 \mathrm{C}$ & 109.5 & $\mathrm{H} 11 \mathrm{H}-\mathrm{C} 11 \mathrm{C}-\mathrm{H} 11 \mathrm{I}$ & 109.5 \\
\hline $\mathrm{C} 10 \mathrm{~A}-\mathrm{C} 12 \mathrm{~A}-\mathrm{H} 12 \mathrm{~A}$ & 109.5 & $\mathrm{C} 10 \mathrm{C}-\mathrm{C} 12 \mathrm{C}-\mathrm{H} 12 \mathrm{G}$ & 109.5 \\
\hline $\mathrm{C} 10 \mathrm{~A}-\mathrm{C} 12 \mathrm{~A}-\mathrm{H} 12 \mathrm{~B}$ & 109.5 & $\mathrm{C} 10 \mathrm{C}-\mathrm{C} 12 \mathrm{C}-\mathrm{H} 12 \mathrm{H}$ & 109.5 \\
\hline $\mathrm{H} 12 \mathrm{~A}-\mathrm{C} 12 \mathrm{~A}-\mathrm{H} 12 \mathrm{~B}$ & 109.5 & $\mathrm{H} 12 \mathrm{G}-\mathrm{C} 12 \mathrm{C}-\mathrm{H} 12 \mathrm{H}$ & 109.5 \\
\hline $\mathrm{C} 10 \mathrm{~A}-\mathrm{C} 12 \mathrm{~A}-\mathrm{H} 12 \mathrm{C}$ & 109.5 & $\mathrm{C} 10 \mathrm{C}-\mathrm{C} 12 \mathrm{C}-\mathrm{H} 12 \mathrm{I}$ & 109.5 \\
\hline $\mathrm{H} 12 \mathrm{~A}-\mathrm{C} 12 \mathrm{~A}-\mathrm{H} 12 \mathrm{C}$ & 109.5 & $\mathrm{H} 12 \mathrm{G}-\mathrm{C} 12 \mathrm{C}-\mathrm{H} 12 \mathrm{I}$ & 109.5 \\
\hline $\mathrm{H} 12 \mathrm{~B}-\mathrm{C} 12 \mathrm{~A}-\mathrm{H} 12 \mathrm{C}$ & 109.5 & $\mathrm{H} 12 \mathrm{H}-\mathrm{C} 12 \mathrm{C}-\mathrm{H} 12 \mathrm{I}$ & 109.5 \\
\hline $\mathrm{C} 7 \mathrm{~B}-\mathrm{N} 1 \mathrm{~B}-\mathrm{C} 6 \mathrm{~B}$ & $130.8(2)$ & $\mathrm{C} 7 \mathrm{D}-\mathrm{N} 1 \mathrm{D}-\mathrm{C} 6 \mathrm{D}$ & $130.6(3)$ \\
\hline $\mathrm{C} 7 \mathrm{~B}-\mathrm{N} 1 \mathrm{~B}-\mathrm{H} 1 \mathrm{BB}$ & $106.7(18)$ & $\mathrm{C} 7 \mathrm{D}-\mathrm{N} 1 \mathrm{D}-\mathrm{H} 1 \mathrm{DB}$ & $108.0(17)$ \\
\hline $\mathrm{C} 6 \mathrm{~B}-\mathrm{N} 1 \mathrm{~B}-\mathrm{H} 1 \mathrm{BB}$ & $122.1(17)$ & $\mathrm{C} 6 \mathrm{D}-\mathrm{N} 1 \mathrm{D}-\mathrm{H} 1 \mathrm{DB}$ & $121.4(17)$ \\
\hline $\mathrm{C} 7 \mathrm{~B}-\mathrm{N} 2 \mathrm{~B}-\mathrm{C} 8 \mathrm{~B}$ & $115.8(2)$ & $\mathrm{C} 7 \mathrm{D}-\mathrm{N} 2 \mathrm{D}-\mathrm{C} 8 \mathrm{D}$ & $115.7(2)$ \\
\hline $\mathrm{C} 10 \mathrm{~B}-\mathrm{N} 3 \mathrm{~B}-\mathrm{C} 7 \mathrm{~B}$ & $116.6(2)$ & $\mathrm{C} 10 \mathrm{D}-\mathrm{N} 3 \mathrm{D}-\mathrm{C} 7 \mathrm{D}$ & $116.9(2)$ \\
\hline $\mathrm{C} 2 \mathrm{~B}-\mathrm{C} 1 \mathrm{~B}-\mathrm{C} 6 \mathrm{~B}$ & $120.7(3)$ & $\mathrm{C} 2 \mathrm{D}-\mathrm{C} 1 \mathrm{D}-\mathrm{C} 6 \mathrm{D}$ & 120.5 \\
\hline $\mathrm{C} 2 \mathrm{~B}-\mathrm{C} 1 \mathrm{~B}-\mathrm{H} 1 \mathrm{BA}$ & 119.6 & $\mathrm{C} 2 \mathrm{D}-\mathrm{C} 1 \mathrm{D}-\mathrm{H} 1 \mathrm{DA}$ & 119.8 \\
\hline $\mathrm{C} 6 \mathrm{~B}-\mathrm{C} 1 \mathrm{~B}-\mathrm{H} 1 \mathrm{BA}$ & 119.6 & $\mathrm{C} 6 \mathrm{D}-\mathrm{C} 1 \mathrm{D}-\mathrm{H} 1 \mathrm{DA}$ & 119.8 \\
\hline $\mathrm{C} 1 \mathrm{~B}-\mathrm{C} 2 \mathrm{~B}-\mathrm{C} 3 \mathrm{~B}$ & $120.3(3)$ & $\mathrm{C} 1 \mathrm{D}-\mathrm{C} 2 \mathrm{D}-\mathrm{C} 3 \mathrm{D}$ & $120.8(3)$ \\
\hline $\mathrm{C} 1 \mathrm{~B}-\mathrm{C} 2 \mathrm{~B}-\mathrm{H} 2 \mathrm{BA}$ & 119.9 & $\mathrm{C} 1 \mathrm{D}-\mathrm{C} 2 \mathrm{D}-\mathrm{H} 2 \mathrm{DA}$ & 119.6 \\
\hline $\mathrm{C} 3 \mathrm{~B}-\mathrm{C} 2 \mathrm{~B}-\mathrm{H} 2 \mathrm{BA}$ & 119.9 & $\mathrm{C} 3 \mathrm{D}-\mathrm{C} 2 \mathrm{D}-\mathrm{H} 2 \mathrm{DA}$ & 119.6 \\
\hline $\mathrm{C} 4 \mathrm{~B}-\mathrm{C} 3 \mathrm{~B}-\mathrm{C} 2 \mathrm{~B}$ & $119.3(3)$ & $\mathrm{C} 4 \mathrm{D}-\mathrm{C} 3 \mathrm{D}-\mathrm{C} 2 \mathrm{D}$ & $118.7(3)$ \\
\hline $\mathrm{C} 4 \mathrm{~B}-\mathrm{C} 3 \mathrm{~B}-\mathrm{H} 3 \mathrm{BA}$ & 120.4 & $\mathrm{C} 4 \mathrm{D}-\mathrm{C} 3 \mathrm{D}-\mathrm{H} 3 \mathrm{DA}$ & 120.6 \\
\hline $\mathrm{C} 2 \mathrm{~B}-\mathrm{C} 3 \mathrm{~B}-\mathrm{H} 3 \mathrm{BA}$ & 120.4 & $\mathrm{C} 2 \mathrm{D}-\mathrm{C} 3 \mathrm{D}-\mathrm{H} 3 \mathrm{DA}$ & 120.6 \\
\hline $\mathrm{C} 3 \mathrm{~B}-\mathrm{C} 4 \mathrm{~B}-\mathrm{C} 5 \mathrm{~B}$ & $120.9(3)$ & $\mathrm{C} 3 \mathrm{D}-\mathrm{C} 4 \mathrm{D}-\mathrm{C} 5 \mathrm{D}$ & $121.6(3)$ \\
\hline $\mathrm{C} 3 \mathrm{~B}-\mathrm{C} 4 \mathrm{~B}-\mathrm{H} 4 \mathrm{BA}$ & 119.5 & $\mathrm{C} 3 \mathrm{D}-\mathrm{C} 4 \mathrm{D}-\mathrm{H} 4 \mathrm{DA}$ & 119.2 \\
\hline $\mathrm{C} 5 \mathrm{~B}-\mathrm{C} 4 \mathrm{~B}-\mathrm{H} 4 \mathrm{BA}$ & 119.5 & $\mathrm{C} 5 \mathrm{D}-\mathrm{C} 4 \mathrm{D}-\mathrm{H} 4 \mathrm{DA}$ & 119.2 \\
\hline $\mathrm{C} 6 \mathrm{~B}-\mathrm{C} 5 \mathrm{~B}-\mathrm{C} 4 \mathrm{~B}$ & $120.0(2)$ & $\mathrm{C} 4 \mathrm{D}-\mathrm{C} 5 \mathrm{D}-\mathrm{C} 6 \mathrm{D}$ & $119.4(2)$ \\
\hline $\mathrm{C} 6 \mathrm{~B}-\mathrm{C} 5 \mathrm{~B}-\mathrm{H} 5 \mathrm{BA}$ & 120.0 & $\mathrm{C} 4 \mathrm{D}-\mathrm{C} 5 \mathrm{D}-\mathrm{H} 5 \mathrm{DA}$ & 120.3 \\
\hline $\mathrm{C} 4 \mathrm{~B}-\mathrm{C} 5 \mathrm{~B}-\mathrm{H} 5 \mathrm{BA}$ & 120.0 & $\mathrm{C} 6 \mathrm{D}-\mathrm{C} 5 \mathrm{D}-\mathrm{H} 5 \mathrm{DA}$ & 120.3 \\
\hline $\mathrm{C} 5 \mathrm{~B}-\mathrm{C} 6 \mathrm{~B}-\mathrm{C} 1 \mathrm{~B}$ & $118.8(3)$ & $\mathrm{C} 5 \mathrm{D}-\mathrm{C} 6 \mathrm{D}-\mathrm{C} 1 \mathrm{D}$ & $119.0(2)$ \\
\hline $\mathrm{C} 5 \mathrm{~B}-\mathrm{C} 6 \mathrm{~B}-\mathrm{N} 1 \mathrm{~B}$ & $124.8(2)$ & $\mathrm{C} 5 \mathrm{D}-\mathrm{C} 6 \mathrm{D}-\mathrm{N} 1 \mathrm{D}$ & $124.6(2)$ \\
\hline $\mathrm{C} 1 \mathrm{~B}-\mathrm{C} 6 \mathrm{~B}-\mathrm{N} 1 \mathrm{~B}$ & $116.4(2)$ & $\mathrm{C} 1 \mathrm{D}-\mathrm{C} 6 \mathrm{D}-\mathrm{N} 1 \mathrm{D}$ & $116.5(2)$ \\
\hline $\mathrm{N} 2 \mathrm{~B}-\mathrm{C} 7 \mathrm{~B}-\mathrm{N} 3 \mathrm{~B}$ & $126.7(2)$ & $\mathrm{N} 2 \mathrm{D}-\mathrm{C} 7 \mathrm{D}-\mathrm{N} 3 \mathrm{D}$ & $126.3(2)$ \\
\hline $\mathrm{N} 2 \mathrm{~B}-\mathrm{C} 7 \mathrm{~B}-\mathrm{N} 1 \mathrm{~B}$ & $120.6(3)$ & $\mathrm{N} 2 \mathrm{D}-\mathrm{C} 7 \mathrm{D}-\mathrm{N} 1 \mathrm{D}$ & $120.6(3)$ \\
\hline $\mathrm{N} 3 \mathrm{~B}-\mathrm{C} 7 \mathrm{~B}-\mathrm{N} 1 \mathrm{~B}$ & $112.7(2)$ & $\mathrm{N} 3 \mathrm{D}-\mathrm{C} 7 \mathrm{D}-\mathrm{N} 1 \mathrm{D}$ & $113.0(2)$ \\
\hline $\mathrm{N} 2 \mathrm{~B}-\mathrm{C} 8 \mathrm{~B}-\mathrm{C} 9 \mathrm{~B}$ & $121.3(2)$ & $\mathrm{N} 2 \mathrm{D}-\mathrm{C} 8 \mathrm{D}-\mathrm{C} 9 \mathrm{D}$ & $121.8(2)$ \\
\hline $\mathrm{N} 2 \mathrm{~B}-\mathrm{C} 8 \mathrm{~B}-\mathrm{C} 11 \mathrm{~B}$ & $116.6(2)$ & $\mathrm{N} 2 \mathrm{D}-\mathrm{C} 8 \mathrm{D}-\mathrm{C} 11 \mathrm{D}$ & $116.0(3)$ \\
\hline $\mathrm{C} 9 \mathrm{~B}-\mathrm{C} 8 \mathrm{~B}-\mathrm{C} 11 \mathrm{~B}$ & $122.1(2)$ & $\mathrm{C} 9 \mathrm{D}-\mathrm{C} 8 \mathrm{D}-\mathrm{C} 11 \mathrm{D}$ & $122.2(2)$ \\
\hline $\mathrm{C} 8 \mathrm{~B}-\mathrm{C} 9 \mathrm{~B}-\mathrm{C} 10 \mathrm{~B}$ & $118.7(2)$ & $\mathrm{C} 8 \mathrm{D}-\mathrm{C} 9 \mathrm{D}-\mathrm{C} 10 \mathrm{D}$ & $118.5(2)$ \\
\hline $\mathrm{C} 8 \mathrm{~B}-\mathrm{C} 9 \mathrm{~B}-\mathrm{H} 9 \mathrm{BA}$ & 120.6 & $\mathrm{C} 8 \mathrm{D}-\mathrm{C} 9 \mathrm{D}-\mathrm{H} 9 \mathrm{DA}$ & 120.7 \\
\hline $\mathrm{C} 10 \mathrm{~B}-\mathrm{C} 9 \mathrm{~B}-\mathrm{H} 9 \mathrm{BA}$ & 120.6 & $\mathrm{C} 10 \mathrm{D}-\mathrm{C} 9 \mathrm{D}-\mathrm{H} 9 \mathrm{DA}$ & 120.7 \\
\hline $\mathrm{N} 3 \mathrm{~B}-\mathrm{C} 10 \mathrm{~B}-\mathrm{C} 9 \mathrm{~B}$ & $120.9(3)$ & $\mathrm{N} 3 \mathrm{D}-\mathrm{C} 10 \mathrm{D}-\mathrm{C} 9 \mathrm{D}$ & $120.7(3)$ \\
\hline $\mathrm{N} 3 \mathrm{~B}-\mathrm{C} 10 \mathrm{~B}-\mathrm{C} 12 \mathrm{~B}$ & $117.3(2)$ & $\mathrm{N} 3 \mathrm{D}-\mathrm{C} 10 \mathrm{D}-\mathrm{C} 12 \mathrm{D}$ & $117.1(2)$ \\
\hline $\mathrm{C} 9 \mathrm{~B}-\mathrm{C} 10 \mathrm{~B}-\mathrm{C} 12 \mathrm{~B}$ & $121.8(2)$ & $\mathrm{C} 9 \mathrm{D}-\mathrm{C} 10 \mathrm{D}-\mathrm{C} 12 \mathrm{D}$ & $122.2(2)$ \\
\hline $\mathrm{C} 8 \mathrm{~B}-\mathrm{C} 11 \mathrm{~B}-\mathrm{H} 11 \mathrm{D}$ & 109.5 & $\mathrm{C} 8 \mathrm{D}-\mathrm{C} 11 \mathrm{D}-\mathrm{H} 11 \mathrm{~J}$ & 109.5 \\
\hline $\mathrm{C} 8 \mathrm{~B}-\mathrm{C} 11 \mathrm{~B}-\mathrm{H} 11 \mathrm{E}$ & 109.5 & $\mathrm{C} 8 \mathrm{D}-\mathrm{C} 11 \mathrm{D}-\mathrm{H} 11 \mathrm{~K}$ & 109.5 \\
\hline $\mathrm{H} 11 \mathrm{D}-\mathrm{C} 11 \mathrm{~B}-\mathrm{H} 11 \mathrm{E}$ & 109.5 & $\mathrm{H} 11 \mathrm{~J}-\mathrm{C} 11 \mathrm{D}-\mathrm{H} 11 \mathrm{~K}$ & 109.5 \\
\hline $\mathrm{C} 8 \mathrm{~B}-\mathrm{C} 11 \mathrm{~B}-\mathrm{H} 11 \mathrm{~F}$ & 109.5 & $\mathrm{C} 8 \mathrm{D}-\mathrm{C} 11 \mathrm{D}-\mathrm{H} 11 \mathrm{~L}$ & 109.5 \\
\hline $\mathrm{H} 11 \mathrm{D}-\mathrm{C} 11 \mathrm{~B}-\mathrm{H} 11 \mathrm{~F}$ & 109.5 & $\mathrm{H} 11 \mathrm{~J}-\mathrm{C} 11 \mathrm{D}-\mathrm{H} 11 \mathrm{~L}$ & 109.5 \\
\hline $\mathrm{H} 11 \mathrm{E}-\mathrm{C} 11 \mathrm{~B}-\mathrm{H} 11 \mathrm{~F}$ & 109.5 & $\mathrm{H} 11 \mathrm{~K}-\mathrm{C} 11 \mathrm{D}-\mathrm{H} 11 \mathrm{~L}$ & 109.5 \\
\hline
\end{tabular}




\begin{tabular}{|c|c|c|c|}
\hline $\mathrm{C} 10 \mathrm{~B}-\mathrm{C} 12 \mathrm{~B}-\mathrm{H} 12 \mathrm{D}$ & 109.5 & $\mathrm{C} 10 \mathrm{D}-\mathrm{C} 12 \mathrm{D}-\mathrm{H} 12 \mathrm{~J}$ & 109.5 \\
\hline $\mathrm{C} 10 \mathrm{~B}-\mathrm{C} 12 \mathrm{~B}-\mathrm{H} 12 \mathrm{E}$ & 109.5 & $\mathrm{C} 10 \mathrm{D}-\mathrm{C} 12 \mathrm{D}-\mathrm{H} 12 \mathrm{~K}$ & 109.5 \\
\hline $\mathrm{H} 12 \mathrm{D}-\mathrm{C} 12 \mathrm{~B}-\mathrm{H} 12 \mathrm{E}$ & 109.5 & $\mathrm{H} 12 \mathrm{~J}-\mathrm{C} 12 \mathrm{D}-\mathrm{H} 12 \mathrm{~K}$ & 109.5 \\
\hline $\mathrm{C} 10 \mathrm{~B}-\mathrm{C} 12 \mathrm{~B}-\mathrm{H} 12 \mathrm{~F}$ & 109.5 & $\mathrm{C} 10 \mathrm{D}-\mathrm{C} 12 \mathrm{D}-\mathrm{H} 12 \mathrm{~L}$ & 109.5 \\
\hline $\mathrm{H} 12 \mathrm{D}-\mathrm{C} 12 \mathrm{~B}-\mathrm{H} 12 \mathrm{~F}$ & 109.5 & $\mathrm{H} 12 \mathrm{~J}-\mathrm{C} 12 \mathrm{D}-\mathrm{H} 12 \mathrm{~L}$ & 109.5 \\
\hline $\mathrm{H} 12 \mathrm{E}-\mathrm{C} 12 \mathrm{~B}-\mathrm{H} 12 \mathrm{~F}$ & 109.5 & $\mathrm{H} 12 \mathrm{~K}-\mathrm{C} 12 \mathrm{D}-\mathrm{H} 12 \mathrm{~L}$ & 109.5 \\
\hline $\mathrm{C} 6 \mathrm{~A}-\mathrm{C} 1 \mathrm{~A}-\mathrm{C} 2 \mathrm{~A}-\mathrm{C} 3 \mathrm{~A}$ & $0.4(4)$ & $\mathrm{C} 6 \mathrm{C}-\mathrm{C} 1 \mathrm{C}-\mathrm{C} 2 \mathrm{C}-\mathrm{C} 3 \mathrm{C}$ & $0.3(4)$ \\
\hline $\mathrm{C} 1 \mathrm{~A}-\mathrm{C} 2 \mathrm{~A}-\mathrm{C} 3 \mathrm{~A}-\mathrm{C} 4 \mathrm{~A}$ & $0.1(4)$ & $\mathrm{C} 1 \mathrm{C}-\mathrm{C} 2 \mathrm{C}-\mathrm{C} 3 \mathrm{C}-\mathrm{C} 4 \mathrm{C}$ & $-1.2(4)$ \\
\hline $\mathrm{C} 2 \mathrm{~A}-\mathrm{C} 3 \mathrm{~A}-\mathrm{C} 4 \mathrm{~A}-\mathrm{C} 5 \mathrm{~A}$ & $-0.3(4)$ & $\mathrm{C} 2 \mathrm{C}-\mathrm{C} 3 \mathrm{C}-\mathrm{C} 4 \mathrm{C}-\mathrm{C} 5 \mathrm{C}$ & $1.7(4)$ \\
\hline $\mathrm{C} 3 \mathrm{~A}-\mathrm{C} 4 \mathrm{~A}-\mathrm{C} 5 \mathrm{~A}-\mathrm{C} 6 \mathrm{~A}$ & $0.2(4)$ & $\mathrm{C} 3 \mathrm{C}-\mathrm{C} 4 \mathrm{C}-\mathrm{C} 5 \mathrm{C}-\mathrm{C} 6 \mathrm{C}$ & $-1.2(4)$ \\
\hline $\mathrm{C} 4 \mathrm{~A}-\mathrm{C} 5 \mathrm{~A}-\mathrm{C} 6 \mathrm{~A}-\mathrm{C} 1 \mathrm{~A}$ & $0.3(4)$ & $\mathrm{C} 2 \mathrm{C}-\mathrm{C} 1 \mathrm{C}-\mathrm{C} 6 \mathrm{C}-\mathrm{C} 5 \mathrm{C}$ & $0.2(4)$ \\
\hline $\mathrm{C} 4 \mathrm{~A}-\mathrm{C} 5 \mathrm{~A}-\mathrm{C} 6 \mathrm{~A}-\mathrm{N} 1 \mathrm{~A}$ & $-178.5(2)$ & $\mathrm{C} 2 \mathrm{C}-\mathrm{C} 1 \mathrm{C}-\mathrm{C} 6 \mathrm{C}-\mathrm{N} 1 \mathrm{C}$ & $-179.2(2)$ \\
\hline $\mathrm{C} 2 \mathrm{~A}-\mathrm{C} 1 \mathrm{~A}-\mathrm{C} 6 \mathrm{~A}-\mathrm{C} 5 \mathrm{~A}$ & $-0.5(4)$ & $\mathrm{C} 4 \mathrm{C}-\mathrm{C} 5 \mathrm{C}-\mathrm{C} 6 \mathrm{C}-\mathrm{C} 1 \mathrm{C}$ & $0.2(4)$ \\
\hline $\mathrm{C} 2 \mathrm{~A}-\mathrm{C} 1 \mathrm{~A}-\mathrm{C} 6 \mathrm{~A}-\mathrm{N} 1 \mathrm{~A}$ & $178.3(2)$ & $\mathrm{C} 4 \mathrm{C}-\mathrm{C} 5 \mathrm{C}-\mathrm{C} 6 \mathrm{C}-\mathrm{N} 1 \mathrm{C}$ & $179.6(2)$ \\
\hline $\mathrm{C} 7 \mathrm{~A}-\mathrm{N} 1 \mathrm{~A}-\mathrm{C} 6 \mathrm{~A}-\mathrm{C} 5 \mathrm{~A}$ & $9.4(4)$ & $\mathrm{C} 7 \mathrm{C}-\mathrm{N} 1 \mathrm{C}-\mathrm{C} 6 \mathrm{C}-\mathrm{C} 1 \mathrm{C}$ & $173.8(3)$ \\
\hline $\mathrm{C} 7 \mathrm{~A}-\mathrm{N} 1 \mathrm{~A}-\mathrm{C} 6 \mathrm{~A}-\mathrm{C} 1 \mathrm{~A}$ & $-169.3(3)$ & $\mathrm{C} 7 \mathrm{C}-\mathrm{N} 1 \mathrm{C}-\mathrm{C} 6 \mathrm{C}-\mathrm{C} 5 \mathrm{C}$ & $-5.6(4)$ \\
\hline $\mathrm{C} 8 \mathrm{~A}-\mathrm{N} 2 \mathrm{~A}-\mathrm{C} 7 \mathrm{~A}-\mathrm{N} 3 \mathrm{~A}$ & $1.0(4)$ & $\mathrm{C} 8 \mathrm{C}-\mathrm{N} 2 \mathrm{C}-\mathrm{C} 7 \mathrm{C}-\mathrm{N} 3 \mathrm{C}$ & $-3.5(4)$ \\
\hline $\mathrm{C} 8 \mathrm{~A}-\mathrm{N} 2 \mathrm{~A}-\mathrm{C} 7 \mathrm{~A}-\mathrm{N} 1 \mathrm{~A}$ & $-176.9(2)$ & $\mathrm{C} 8 \mathrm{C}-\mathrm{N} 2 \mathrm{C}-\mathrm{C} 7 \mathrm{C}-\mathrm{N} 1 \mathrm{C}$ & $176.3(2)$ \\
\hline $\mathrm{C} 10 \mathrm{~A}-\mathrm{N} 3 \mathrm{~A}-\mathrm{C} 7 \mathrm{~A}-\mathrm{N} 2 \mathrm{~A}$ & $-1.8(4)$ & $\mathrm{C} 10 \mathrm{C}-\mathrm{N} 3 \mathrm{C}-\mathrm{C} 7 \mathrm{C}-\mathrm{N} 2 \mathrm{C}$ & $4.3(4)$ \\
\hline $\mathrm{C} 10 \mathrm{~A}-\mathrm{N} 3 \mathrm{~A}-\mathrm{C} 7 \mathrm{~A}-\mathrm{N} 1 \mathrm{~A}$ & $176.3(2)$ & $\mathrm{C} 10 \mathrm{C}-\mathrm{N} 3 \mathrm{C}-\mathrm{C} 7 \mathrm{C}-\mathrm{N} 1 \mathrm{C}$ & $-175.6(2)$ \\
\hline $\mathrm{C} 6 \mathrm{~A}-\mathrm{N} 1 \mathrm{~A}-\mathrm{C} 7 \mathrm{~A}-\mathrm{N} 2 \mathrm{~A}$ & $-16.4(4)$ & $\mathrm{C} 6 \mathrm{C}-\mathrm{N} 1 \mathrm{C}-\mathrm{C} 7 \mathrm{C}-\mathrm{N} 2 \mathrm{C}$ & $12.0(4)$ \\
\hline $\mathrm{C} 6 \mathrm{~A}-\mathrm{N} 1 \mathrm{~A}-\mathrm{C} 7 \mathrm{~A}-\mathrm{N} 3 \mathrm{~A}$ & $165.3(2)$ & $\mathrm{C} 6 \mathrm{C}-\mathrm{N} 1 \mathrm{C}-\mathrm{C} 7 \mathrm{C}-\mathrm{N} 3 \mathrm{C}$ & $-168.1(2)$ \\
\hline $\mathrm{C} 7 \mathrm{~A}-\mathrm{N} 2 \mathrm{~A}-\mathrm{C} 8 \mathrm{~A}-\mathrm{C} 9 \mathrm{~A}$ & $0.2(3)$ & $\mathrm{C} 7 \mathrm{C}-\mathrm{N} 2 \mathrm{C}-\mathrm{C} 8 \mathrm{C}-\mathrm{C} 9 \mathrm{C}$ & $-0.2(4)$ \\
\hline $\mathrm{C} 7 \mathrm{~A}-\mathrm{N} 2 \mathrm{~A}-\mathrm{C} 8 \mathrm{~A}-\mathrm{C} 11 \mathrm{~A}$ & $178.5(2)$ & $\mathrm{C} 7 \mathrm{C}-\mathrm{N} 2 \mathrm{C}-\mathrm{C} 8 \mathrm{C}-\mathrm{C} 11 \mathrm{C}$ & $-179.7(2)$ \\
\hline $\mathrm{N} 2 \mathrm{~A}-\mathrm{C} 8 \mathrm{~A}-\mathrm{C} 9 \mathrm{~A}-\mathrm{C} 10 \mathrm{~A}$ & $-0.4(4)$ & $\mathrm{N} 2 \mathrm{C}-\mathrm{C} 8 \mathrm{C}-\mathrm{C} 9 \mathrm{C}-\mathrm{C} 10 \mathrm{C}$ & $2.9(4)$ \\
\hline $\mathrm{C} 11 \mathrm{~A}-\mathrm{C} 8 \mathrm{~A}-\mathrm{C} 9 \mathrm{~A}-\mathrm{C} 10 \mathrm{~A}$ & $-178.7(2)$ & $\mathrm{C} 11 \mathrm{C}-\mathrm{C} 8 \mathrm{C}-\mathrm{C} 9 \mathrm{C}-\mathrm{C} 10 \mathrm{C}$ & $-177.7(2)$ \\
\hline $\mathrm{C} 7 \mathrm{~A}-\mathrm{N} 3 \mathrm{~A}-\mathrm{C} 10 \mathrm{~A}-\mathrm{C} 9 \mathrm{~A}$ & $1.4(3)$ & $\mathrm{C} 7 \mathrm{C}-\mathrm{N} 3 \mathrm{C}-\mathrm{C} 10 \mathrm{C}-\mathrm{C} 9 \mathrm{C}$ & $-1.2(3)$ \\
\hline $\mathrm{C} 7 \mathrm{~A}-\mathrm{N} 3 \mathrm{~A}-\mathrm{C} 10 \mathrm{~A}-\mathrm{C} 12 \mathrm{~A}$ & $-178.7(2)$ & $\mathrm{C} 7 \mathrm{C}-\mathrm{N} 3 \mathrm{C}-\mathrm{C} 10 \mathrm{C}-\mathrm{C} 12 \mathrm{C}$ & $179.6(2)$ \\
\hline $\mathrm{C} 8 \mathrm{~A}-\mathrm{C} 9 \mathrm{~A}-\mathrm{C} 10 \mathrm{~A}-\mathrm{N} 3 \mathrm{~A}$ & $-0.4(4)$ & $\mathrm{C} 8 \mathrm{C}-\mathrm{C} 9 \mathrm{C}-\mathrm{C} 10 \mathrm{C}-\mathrm{N} 3 \mathrm{C}$ & $-2.1(4)$ \\
\hline $\mathrm{C} 8 \mathrm{~A}-\mathrm{C} 9 \mathrm{~A}-\mathrm{C} 10 \mathrm{~A}-\mathrm{C} 12 \mathrm{~A}$ & $179.7(2)$ & $\mathrm{C} 8 \mathrm{C}-\mathrm{C} 9 \mathrm{C}-\mathrm{C} 10 \mathrm{C}-\mathrm{C} 12 \mathrm{C}$ & $177.1(2)$ \\
\hline $\mathrm{C} 6 \mathrm{~B}-\mathrm{C} 1 \mathrm{~B}-\mathrm{C} 2 \mathrm{~B}-\mathrm{C} 3 \mathrm{~B}$ & $0.4(4)$ & $\mathrm{C} 6 \mathrm{D}-\mathrm{C} 1 \mathrm{D}-\mathrm{C} 2 \mathrm{D}-\mathrm{C} 3 \mathrm{D}$ & $-1.1(4)$ \\
\hline $\mathrm{C} 1 \mathrm{~B}-\mathrm{C} 2 \mathrm{~B}-\mathrm{C} 3 \mathrm{~B}-\mathrm{C} 4 \mathrm{~B}$ & $0.6(4)$ & $\mathrm{C} 1 \mathrm{D}-\mathrm{C} 2 \mathrm{D}-\mathrm{C} 3 \mathrm{D}-\mathrm{C} 4 \mathrm{D}$ & $-0.4(4)$ \\
\hline $\mathrm{C} 2 \mathrm{~B}-\mathrm{C} 3 \mathrm{~B}-\mathrm{C} 4 \mathrm{~B}-\mathrm{C} 5 \mathrm{~B}$ & $-0.1(4)$ & $\mathrm{C} 2 \mathrm{D}-\mathrm{C} 3 \mathrm{D}-\mathrm{C} 4 \mathrm{D}-\mathrm{C} 5 \mathrm{D}$ & $0.6(4)$ \\
\hline $\mathrm{C} 3 \mathrm{~B}-\mathrm{C} 4 \mathrm{~B}-\mathrm{C} 5 \mathrm{~B}-\mathrm{C} 6 \mathrm{~B}$ & $-1.5(4)$ & $\mathrm{C} 3 \mathrm{D}-\mathrm{C} 4 \mathrm{D}-\mathrm{C} 5 \mathrm{D}-\mathrm{C} 6 \mathrm{D}$ & $0.9(4)$ \\
\hline $\mathrm{C} 4 \mathrm{~B}-\mathrm{C} 5 \mathrm{~B}-\mathrm{C} 6 \mathrm{~B}-\mathrm{C} 1 \mathrm{~B}$ & $2.5(4)$ & $\mathrm{C} 4 \mathrm{D}-\mathrm{C} 5 \mathrm{D}-\mathrm{C} 6 \mathrm{D}-\mathrm{C} 1 \mathrm{D}$ & $-2.4(4)$ \\
\hline $\mathrm{C} 4 \mathrm{~B}-\mathrm{C} 5 \mathrm{~B}-\mathrm{C} 6 \mathrm{~B}-\mathrm{N} 1 \mathrm{~B}$ & $-177.4(2)$ & $\mathrm{C} 4 \mathrm{D}-\mathrm{C} 5 \mathrm{D}-\mathrm{C} 6 \mathrm{D}-\mathrm{N} 1 \mathrm{D}$ & $178.1(2)$ \\
\hline $\mathrm{C} 2 \mathrm{~B}-\mathrm{C} 1 \mathrm{~B}-\mathrm{C} 6 \mathrm{~B}-\mathrm{C} 5 \mathrm{~B}$ & $-1.9(4)$ & $\mathrm{C} 2 \mathrm{D}-\mathrm{C} 1 \mathrm{D}-\mathrm{C} 6 \mathrm{D}-\mathrm{C} 5 \mathrm{D}$ & $2.5(4)$ \\
\hline $\mathrm{C} 2 \mathrm{~B}-\mathrm{C} 1 \mathrm{~B}-\mathrm{C} 6 \mathrm{~B}-\mathrm{N} 1 \mathrm{~B}$ & $177.9(2)$ & $\mathrm{C} 2 \mathrm{D}-\mathrm{C} 1 \mathrm{D}-\mathrm{C} 6 \mathrm{D}-\mathrm{N} 1 \mathrm{D}$ & $-177.9(2)$ \\
\hline $\mathrm{C} 7 \mathrm{~B}-\mathrm{N} 1 \mathrm{~B}-\mathrm{C} 6 \mathrm{~B}-\mathrm{C} 5 \mathrm{~B}$ & $20.7(4)$ & $\mathrm{C} 7 \mathrm{D}-\mathrm{N} 1 \mathrm{D}-\mathrm{C} 6 \mathrm{D}-\mathrm{C} 5 \mathrm{D}$ & $-21.4(4)$ \\
\hline $\mathrm{C} 7 \mathrm{~B}-\mathrm{N} 1 \mathrm{~B}-\mathrm{C} 6 \mathrm{~B}-\mathrm{C} 1 \mathrm{~B}$ & $-159.1(2)$ & $\mathrm{C} 7 \mathrm{D}-\mathrm{N} 1 \mathrm{D}-\mathrm{C} 6 \mathrm{D}-\mathrm{C} 1 \mathrm{D}$ & $159.0(2)$ \\
\hline $\mathrm{C} 8 \mathrm{~B}-\mathrm{N} 2 \mathrm{~B}-\mathrm{C} 7 \mathrm{~B}-\mathrm{N} 3 \mathrm{~B}$ & $1.1(4)$ & $\mathrm{C} 8 \mathrm{D}-\mathrm{N} 2 \mathrm{D}-\mathrm{C} 7 \mathrm{D}-\mathrm{N} 3 \mathrm{D}$ & $-2.2(4)$ \\
\hline $\mathrm{C} 8 \mathrm{~B}-\mathrm{N} 2 \mathrm{~B}-\mathrm{C} 7 \mathrm{~B}-\mathrm{N} 1 \mathrm{~B}$ & $-177.7(2)$ & $\mathrm{C} 8 \mathrm{D}-\mathrm{N} 2 \mathrm{D}-\mathrm{C} 7 \mathrm{D}-\mathrm{N} 1 \mathrm{D}$ & $176.3(2)$ \\
\hline $\mathrm{C} 10 \mathrm{~B}-\mathrm{N} 3 \mathrm{~B}-\mathrm{C} 7 \mathrm{~B}-\mathrm{N} 2 \mathrm{~B}$ & $-2.7(4)$ & $\mathrm{C} 10 \mathrm{D}-\mathrm{N} 3 \mathrm{D}-\mathrm{C} 7 \mathrm{D}-\mathrm{N} 2 \mathrm{D}$ & $2.9(4)$ \\
\hline $\mathrm{C} 10 \mathrm{~B}-\mathrm{N} 3 \mathrm{~B}-\mathrm{C} 7 \mathrm{~B}-\mathrm{N} 1 \mathrm{~B}$ & $176.2(2)$ & $\mathrm{C} 10 \mathrm{D}-\mathrm{N} 3 \mathrm{D}-\mathrm{C} 7 \mathrm{D}-\mathrm{N} 1 \mathrm{D}$ & $-175.6(2)$ \\
\hline $\mathrm{C} 6 \mathrm{~B}-\mathrm{N} 1 \mathrm{~B}-\mathrm{C} 7 \mathrm{~B}-\mathrm{N} 2 \mathrm{~B}$ & $-9.0(4)$ & $\mathrm{C} 6 \mathrm{D}-\mathrm{N} 1 \mathrm{D}-\mathrm{C} 7 \mathrm{D}-\mathrm{N} 2 \mathrm{D}$ & $13.2(4)$ \\
\hline $\mathrm{C} 6 \mathrm{~B}-\mathrm{N} 1 \mathrm{~B}-\mathrm{C} 7 \mathrm{~B}-\mathrm{N} 3 \mathrm{~B}$ & $172.0(2)$ & $\mathrm{C} 6 \mathrm{D}-\mathrm{N} 1 \mathrm{D}-\mathrm{C} 7 \mathrm{D}-\mathrm{N} 3 \mathrm{D}$ & $-168.1(2)$ \\
\hline $\mathrm{C} 7 \mathrm{~B}-\mathrm{N} 2 \mathrm{~B}-\mathrm{C} 8 \mathrm{~B}-\mathrm{C} 9 \mathrm{~B}$ & $1.4(3)$ & $\mathrm{C} 7 \mathrm{D}-\mathrm{N} 2 \mathrm{D}-\mathrm{C} 8 \mathrm{D}-\mathrm{C} 9 \mathrm{D}$ & $-0.5(4)$ \\
\hline
\end{tabular}




$\begin{array}{llll}\mathrm{C} 7 \mathrm{~B}-\mathrm{N} 2 \mathrm{~B}-\mathrm{C} 8 \mathrm{~B}-\mathrm{C} 11 \mathrm{~B} & -179.2(2) & \mathrm{C} 7 \mathrm{D}-\mathrm{N} 2 \mathrm{D}-\mathrm{C} 8 \mathrm{D}-\mathrm{C} 11 \mathrm{D} & -179.7(2) \\ \mathrm{N} 2 \mathrm{~B}-\mathrm{C} 8 \mathrm{~B}-\mathrm{C} 9 \mathrm{~B}-\mathrm{C} 10 \mathrm{~B} & -2.2(4) & \mathrm{N} 2 \mathrm{D}-\mathrm{C} 8 \mathrm{D}-\mathrm{C} 9 \mathrm{D}-\mathrm{C} 10 \mathrm{D} & 2.1(4) \\ \mathrm{C} 11 \mathrm{~B}-\mathrm{C} 8 \mathrm{~B}-\mathrm{C} 9 \mathrm{~B}-\mathrm{C} 10 \mathrm{~B} & 178.5(2) & \mathrm{C} 11 \mathrm{D}-\mathrm{C} 8 \mathrm{D}-\mathrm{C} 9 \mathrm{D}-\mathrm{C} 10 \mathrm{D} & -178.7(2) \\ \mathrm{C} 7 \mathrm{~B}-\mathrm{N} 3 \mathrm{~B}-\mathrm{C} 10 \mathrm{~B}-\mathrm{C} 9 \mathrm{~B} & 1.8(3) & \mathrm{C} 7 \mathrm{D}-\mathrm{N} 3 \mathrm{D}-\mathrm{C} 10 \mathrm{D}-\mathrm{C} 9 \mathrm{D} & -1.1(3) \\ \mathrm{C} 7 \mathrm{~B}-\mathrm{N} 3 \mathrm{~B}-\mathrm{C} 10 \mathrm{~B}-\mathrm{C} 12 \mathrm{~B} & -176.9(2) & \mathrm{C} 7 \mathrm{D}-\mathrm{N} 3 \mathrm{D}-\mathrm{C} 10 \mathrm{D}-\mathrm{C} 12 \mathrm{D} & 177.2(2) \\ \mathrm{C} 8 \mathrm{~B}-\mathrm{C} 9 \mathrm{~B}-\mathrm{C} 10 \mathrm{~B}-\mathrm{N} 3 \mathrm{~B} & 0.5(4) & \mathrm{C} 8 \mathrm{D}-\mathrm{C} 9 \mathrm{D}-\mathrm{C} 10 \mathrm{D}-\mathrm{N} 3 \mathrm{D} & -1.3(4) \\ \mathrm{C} 8 \mathrm{~B}-\mathrm{C} 9 \mathrm{~B}-\mathrm{C} 10 \mathrm{~B}-\mathrm{C} 12 \mathrm{~B} & 179.1(2) & \mathrm{C} 8 \mathrm{D}-\mathrm{C} 9 \mathrm{D}-\mathrm{C} 10 \mathrm{D}-\mathrm{C} 12 \mathrm{D} & -179.5(2)\end{array}$

Hydrogen-bond geometry $\left(A,{ }^{\circ}\right)$

\begin{tabular}{lllll}
\hline$D-\mathrm{H} \cdots A$ & $D-\mathrm{H}$ & $\mathrm{H} \cdots A$ & $D \cdots A$ & $D-\mathrm{H} \cdots A$ \\
\hline $\mathrm{N} 1 A-\mathrm{H} 1 A B \cdots \mathrm{N} 3 B$ & $0.91(3)$ & $2.11(3)$ & $2.997(3)$ & $165(2)$ \\
$\mathrm{N} 1 B-\mathrm{H} 1 B B \cdots \mathrm{N} 3 A$ & $0.97(3)$ & $2.08(3)$ & $3.022(3)$ & $162(3)$ \\
$\mathrm{N} 1 C-\mathrm{H} 1 C B \cdots \mathrm{N} 3 D$ & $0.94(3)$ & $2.05(3)$ & $2.975(3)$ & $166(3)$ \\
$\mathrm{N} 1 D-\mathrm{H} 1 D B \cdots \mathrm{N} 3 C$ & $0.92(3)$ & $2.08(3)$ & $2.987(3)$ & $167(3)$ \\
\hline
\end{tabular}

